Portland State University

PDXScholar

10-3-1995

\title{
Electrostatic Interactions at Membrane-water Interfaces and Distribution of 2, 4, 6-Trichlorophenol in a Membrane Model System
}

Isolde Sieder

Portland State University

Follow this and additional works at: https://pdxscholar.library.pdx.edu/open_access_etds

Part of the Physics Commons

Let us know how access to this document benefits you.

Recommended Citation

Sieder, Isolde, "Electrostatic Interactions at Membrane-water Interfaces and Distribution of 2, 4, 6-Trichlorophenol in a Membrane Model System" (1995). Dissertations and Theses. Paper 5087. https://doi.org/10.15760/etd.6963

This Thesis is brought to you for free and open access. It has been accepted for inclusion in Dissertations and Theses by an authorized administrator of PDXScholar. Please contact us if we can make this document more accessible: pdxscholar@pdx.edu. 


\section{THESIS APPROVAL}

The abstract and thesis of Isolde Sieder for the Master of Science in Physics were presented October 3,1995, and accepted by the thesis committee and the department.

COMMITTEE APPROVALS:
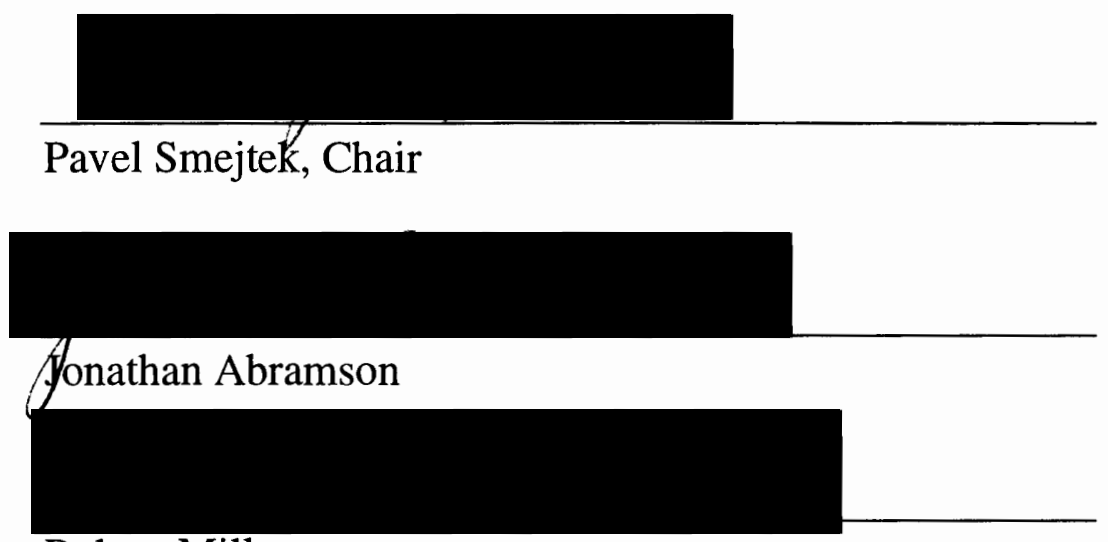

Robert Millette

Representative of the Office of Graduate Studies

DEPARTMENT APPROVAL:

Erik Bodegom, Chair

Department of Physics

$* * * * * * * * * * * * * * * * * * * * * * * * * * * * * * * * * * * * * * * * * * * * * * * * * * * * * * * * * * * * * * * * *$

ACCEPTED FOR PORTLAND STATE UNIVERSITY BY THE LIBARY

by

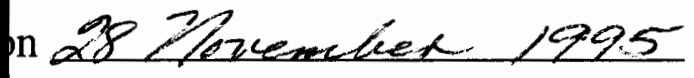




\begin{abstract}
An abstract of the thesis of Isolde Sieder for the Master of Science in Physics presented October 3, 1995.
\end{abstract}

Title: Electrostatic Interactions at Membrane-Water Interfaces and Distribution of 2,4,6-Trichlorophenol in a Membrane Model System

It is generally accepted that biological membranes consist of a lipid bilayer matrix with proteins incorporated into the lipid bilayer. Typically, these membranes are negatively charged due to the presence of negatively charged lipids in the bilayer as well as negatively charged molecular groups on proteins. Biologically active molecules, such as environmental pollutants, enter the membrane from the aqueous phase by adsorption or partitioning into the lipid bilayer. The thesis consists of two parts.

Part I is a computational study of spatial distribution of electric potential in the aqueous portion of the membrane-water interface using two models of charge distribution: (i) the discrete charge model, in which charges are located on a square lattice either on the surface or embedded in the membrane: (ii) the 
continuous charge density (smeared charge), Gouy-Chapman, model in which the charge is assumed to be evenly spread on the membrane surface. The computed distributions of electric potential are used to predict spatial distributions of positively charged hexavalent cation of Ruthenium Red (RuR) at the membranewater interface. It was found that anomalous behavior of RuR cannot be explained by this version of the discrete charge theory.

Part II is concerned with the distribution of ionized and un-ionized species of 2,4,6-Trichlorophenol $(2,4,6-\mathrm{TrCP})$ in octanol-water system, which is often used as an experimental model for predicting the distribution of toxic chemicals in the environment. In this experimental study we obtained the $\mathrm{pH}$ dependence of the total distribution coefficient of 2,4,6-TrCP from which the octanol water partition coefficients of the un-ionized and ionized species were determined. We compared the octanol-water partition coefficient of several chlorophenols with experimental data on adsorption of ionized chlorophenols to lipid membranes. It was found that the membrane-water partition coefficient of ionized $2,4,6-\operatorname{TrCP}$ is about 240 greater than that predicted from the octanol-water system. This finding supports the hypothesis that octanol-water partition coefficients cannot be used for predicting concentrations of ionized species of chlorophenols in lipid membranes. 


\section{ELECTROSTATIC INTERACTIONS AT \\ MEMBRANE-WATER INTERFACES}

AND

DISTRIBUTION OF 2,4,6-TRICHLOROPHENOL

IN A MEMBRANE MODEL SYSTEM

b y

ISOLDE SIEDER

A thesis submitted in partial fulfillment of the requirements for the degree of

MASTER OF SCIENCE

in

PHYSICS

Portland State University

1995 
Für

meine El tern Gretel und Gerhard und mein Bruder Georg 


\section{Acknowledgments}

First and most of all I would like to thank my advisor Prof. Pavel Smejtek. It was a great pleasure to work with him. His excitement about physics, his steady flux in producing physical ideas, and his finding of new papers of interest motivated me to search more and deeper into physics. With his scientific knowledge and humorous personality he helped me to accomplish this work and always created a relaxed working atmosphere.

The experiments would not have been possible without the encouraging help of Prof. Shanru Wang. She taught me all the necessary chemical techniques and was always willing to help me with experimental problems. The availability of the centrifuges in Prof. Bob Millette's lab contributed significantly to finish the experiments on time, thanks to the lab members and their tolerance of the smell of octanol.

I enjoyed working with Piet Schmidt and Rob Ward in our lab. Serious discussions as jokes about our experiments and frustration as well as laughter was always present in our lab. Special thanks to Piet who helped me to overcome my struggles with computers and computer programs.

I also want to thank Prof. Jonathan Abramson who helped me through his biophysics lecture to get started in this field of physics which was totally new for me. I would especially like to thank Prof. Eric Bodegom who found ways to support me financially. I appreciated the help of Margie Fyfield, she helped me overcome my struggles with the English language but, furthermore, motivated me enormous to finish my thesis. She and all my friends, here and in Germany, cheered me up and helped me in my busiest time in so many different 
ways. Thank you all !

Finally I want to thank my parents and my brother very much for their financial and mentally support through this year. I never will forget my great experiences here in the USA ! 


\section{Contents}

Acknowledgments

vi

$\begin{array}{ll}\text { Introduction } & 1\end{array}$

I Electrostatic Interactions

at Membrane-Water Interfaces

1 Theory 5

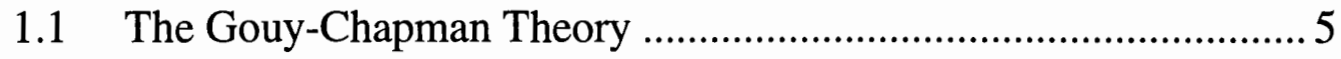

1.2 The Discrete Charge Theory ........................................................ 9

1.3. The Boltzmann Factor.............................................................. 15

2 Electrical Potential and Ion Concentration

Distribution in the Vicinity of the Charged Membrane

Surface

16

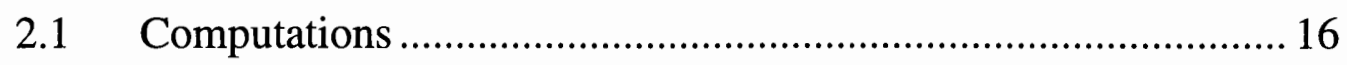

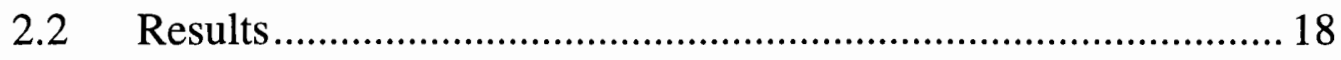




\section{Distribution of 2,4,6-Trichlorophenol in a Membrane Model System}

4.1 Partition of Un-ionized and Ionized Molecules between Water and a Medium of Low Polarity

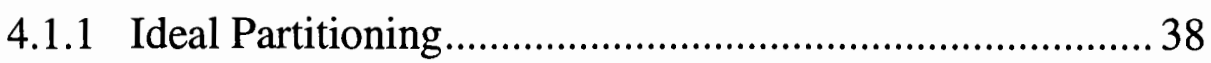

4.1.2 The Octanol-Water System ............................................... 42

a) Description of the Bulk Phases....................................... 42

b) Distribution of Ionized and Un-ionized Species

Between the Two Phases ............................................... 44

4.1.3 The Water-Membrane System ......................................... 46

4.2 Spectrophotometric Measurements.............................................. 48

4.2.1 Lambert-Beer's-Law ....................................................... 49

4.2.2 Solvent Effects ........................................................... 51

$5 \quad$ Materials and Methods $\quad 52$

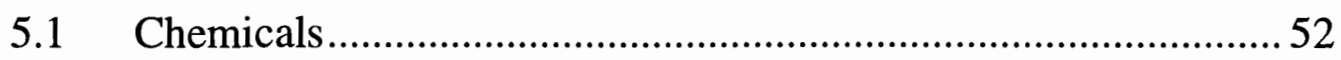

5.2 Instruments and their Specifications .......................................... 52

5.3 Preparation of Octanol and Aqueous Phases ...............................53

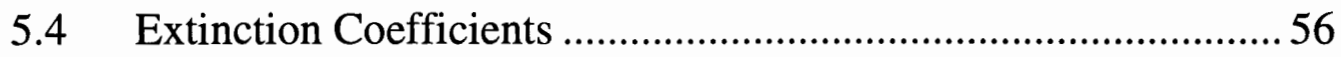

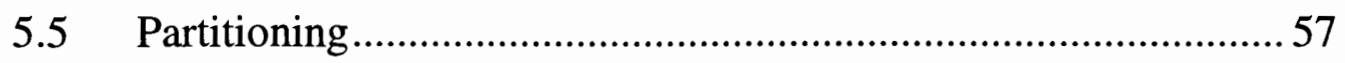


6 Results 59

6.1 Extinction Coefficients .............................................................. 59

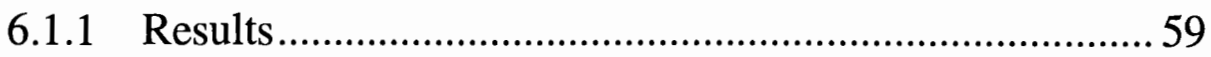

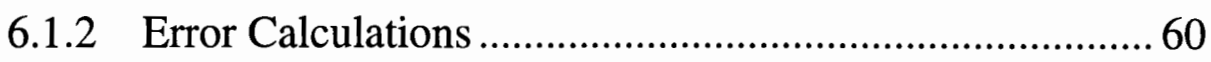

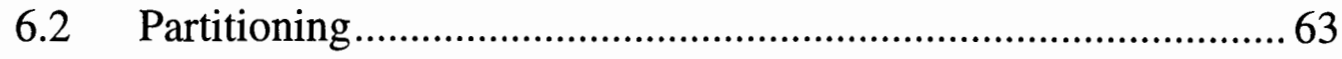

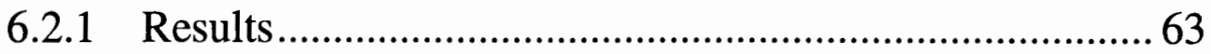

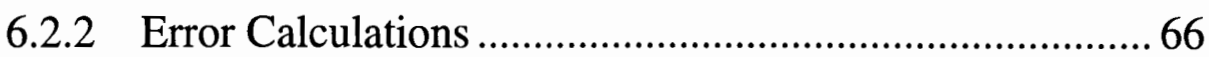

7 Discussion $\quad 72$

7.1 Comparison of the Octanol-Water System with the Membrane-Water System

\section{Appendix A}

Derivations: the Gouy-Chapman Theory

75

\section{Appendix B}

The Partitioning in the Octanol-Water System:

Multicomponent Partitioning 78

References

82 


\section{List of Tables}

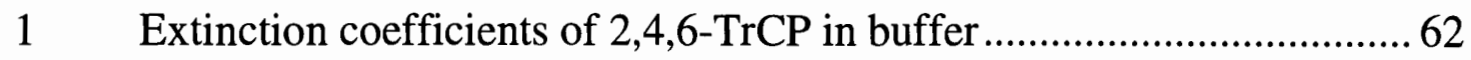

2 Extinction coefficients of 2,4,6-TrCP in water-saturated octanol ..........62

3 Experimental data for the $\mathrm{pH}$ dependence of the total distribution

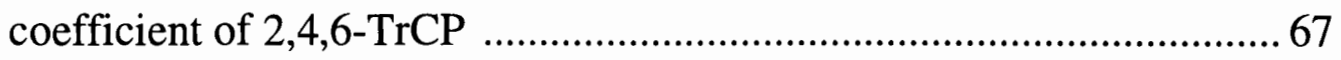

4 Experimental data of the total concentrations of 2,4,6-TrCP in buffer and

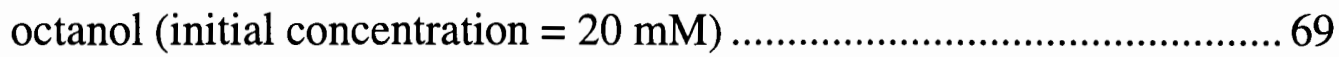

5 Experimental data of the total concentrations of 2,4,6-TrCP in buffer and

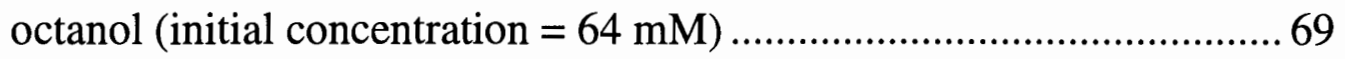

6 Comparison of bulk partition coefficients of three different chlorophenols for lipid-membrane-water and octanol-water systems .... 73 


\section{List of Figures}

1 Conceptual model and molecular structure of phosphatidylcholine ......... 3

2 Fluid mosaic model of a biological membrane ...................................... 3

3 Membrane model used in the Gouy-Chapman theory …........................5

$4 \quad$ Membrane model used in the discrete charge theory ............................ 10

$5 \quad$ Potential profiles for the discrete charge model ................................. 22

$6 \quad$ Concentration profiles for the discrete charge model ........................... 23

7 Potentials as a function of distance from the membrane for the studied

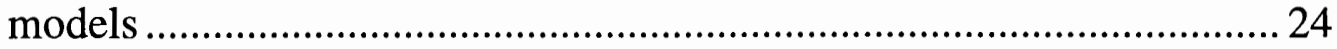

8 Discrete charge potential as a function of embedding depth ................. 25

$9 \quad$ Potentials as a function of surface charge density ............................... 26

10 Boltzmann factors as a function of distance from the membrane........... 27

11 Boltzmann factors for various embedding depth and charge density .....28

12 Molecular structure of 2,4,6-Trichlorophenol ........................................ 30

13 Energy level diagram of a diatomic molecule ......................................49

14 Typical spectrum of the saturated buffer phase …................................55

15 Typical spectrum of the saturated octanol phase …...............................55

16 Spectrum of un-ionized 2,4,6-TrCP in water-saturated octanol ............. 61 
17 Spectrum of ionized 2,4,6-TrCP in octanol-saturated buffer..................61

$18 \mathrm{pH}$ dependence of the total distribution coefficient $\mathrm{D}$ of $2,4,6-\operatorname{TrCP} \ldots . .68$

$19 \mathrm{pH}$ dependence of 2,4,6-TrCP concentrations in buffer and octanol

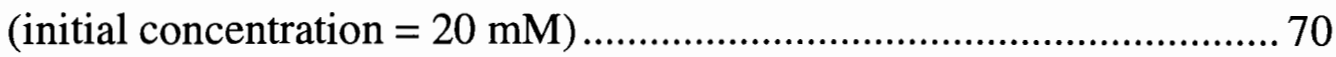

$20 \mathrm{pH}$ dependence of 2,4,6-TrCP concentrations in buffer and octanol

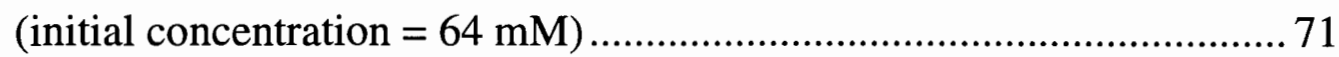




\section{Introduction}

Biological membranes are essential for all forms of life; in addition to separating cells from their environment they give cells their individuality needed to perform specific functions. Biological membranes are highly selective permeability barriers rather than impervious walls (Stryer, 1981). They play an important role in biological communication since they are able to process or generate chemical or electrical signals. Photosynthesis and oxidative phosphorylation, the most important biological energy conversion processes, are also carried out by membranes.

Biological membranes are sheetlike structures which consist mainly of lipids and proteins. The lipids are amphipathic molecules. They contain a hydrophilic headgroup and a hydrophobic $-\left(\mathrm{CH}_{2}\right)$ - chain and can be neutral or charged. The molecular structure of a typical lipid, phosphatidylcholine, is shown in Figure 1. The lipids in the membrane are arranged as a bilayer and provide a suitable environment for proteins embedded in the membrane bilayer. Figure 2 shows the fluid mosaic model of a biological membrane, a model proposed by Singer and Nicolson (1972), generally accepted to describe the gross organization of biological membranes. Membrane proteins perform specific functions, they serve as pumps, gates, receptors, energy transducers, and enzymes (Stryer, 1981). Attempts are being made to understand complex functions of membranes in terms of laws of physics and chemistry.

Lipid bilayer is a structural element common to all biological membranes. This thesis is focused on two aspects of physical properties of lipid bilayers and on the interaction of toxic environmental pollutants with membranes: 
(1) The distribution of electric potential on the aqueous side of membrane water interface in the presence of discrete electric charges at or below the membrane surface. The importance of understanding the electric potential distribution in this region is that it determines the local concentrations of charged molecules at the membrane surface.

(2) The distribution of un-ionized and ionized species of 2,4,6Trichlorophenol between water and octanol. The octanol-water system is frequently used as a simple model to predict the distribution of toxic compounds in environmentally relevant systems. Serious concerns have been raised recently about its applicability, as an experimental model, to predict distributions of ionized toxic compounds between water and lipid membranes. 


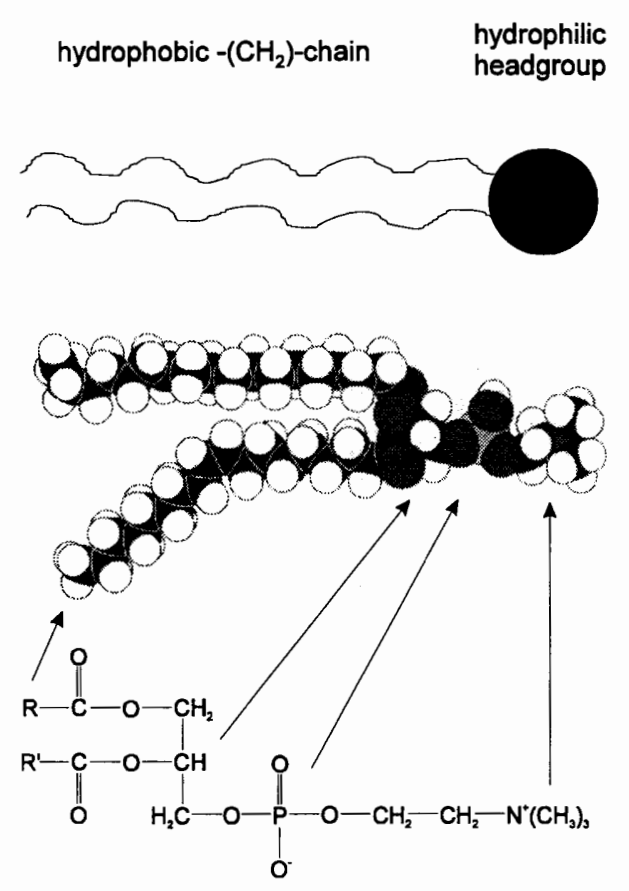

Figure 1. Conceptual model and molecular structure of phosphatidylcholine, a lipid component of all biological membranes.

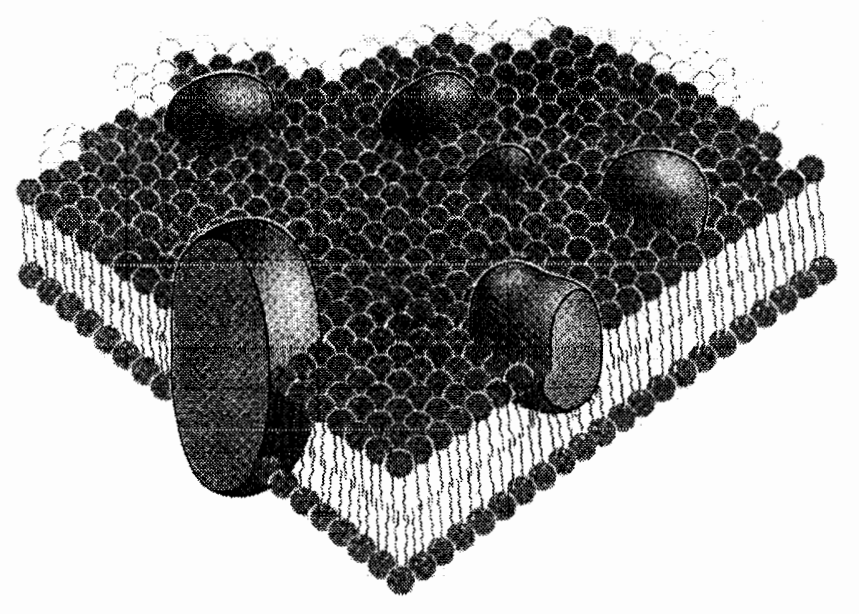

Figure 2. Fluid mosaic model of a biological membrane according to Singer and Nicolson (1972). 


\section{Part I}

\section{Electrostatic Interactions at Membrane- Water Interfaces}




\section{Theory}

\subsection{The Gouy-Chapman Theory}

Gouy (1910) and Chapman (1913) proposed independently the socalled diffuse double layer theory of plane interfaces. Their theory provides an understanding of a variety of electrostatic problems associated with plane interfaces. In the case of the water-membrane interface, the Gouy-Chapman theory offers a good description of the electrostatic interactions between the charged membrane and the ions in the aqueous phase even though it strongly simplifies the

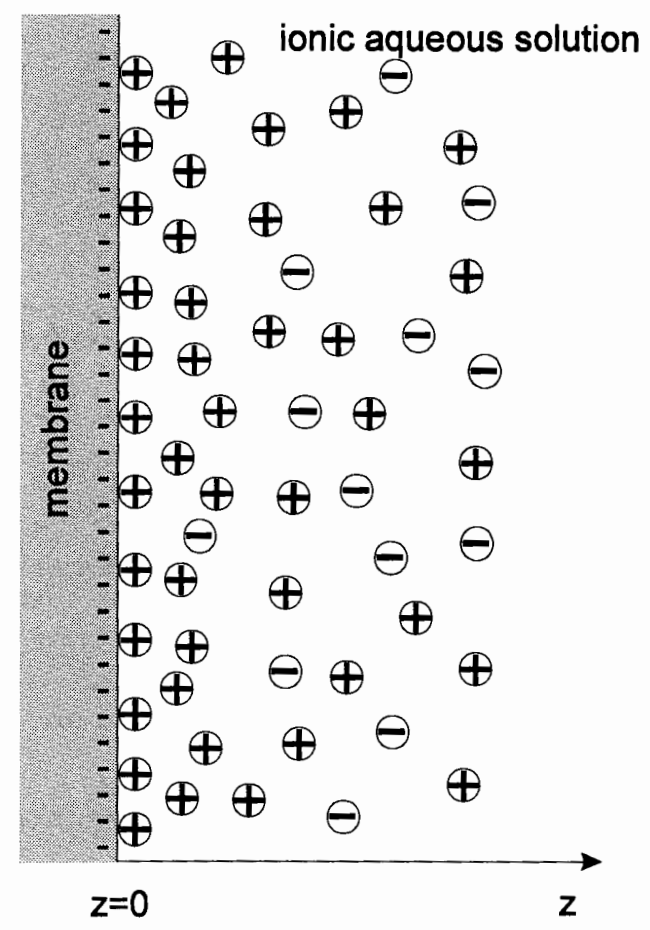

Figure 3. Membrane model used in the Gouy-Chapman theory. real membrane-water interface.

The membrane-water interface is reduced to an infinite plane surface carrying a smeared surface charge which is in contact with the aqueous phase. The ions in the aqueous phase are treated as point charges, and ion-ion interactions, except those affected by the mean potential, are neglected. In Figure 3, the membrane model used for the Gouy-Chapman theory is shown. 
An important feature of this model is the treatment of the three-dimensional membrane-water interface as a one-dimensional system. The Gouy-Chapman theory takes into account only interactions normal to the membrane surface, any interactions parallel to the membrane surface are neglected.

At an infinite distance from the surface the ions in the solution will not feel the repulsive or attractive force of the membrane surface charge, the electrical potential at an infinite distance is zero. But, as the surface is approached, the electric potential, $\psi$, gradually changes. It is assumed that the work required to bring an ion from the bulk of the solution to a point near the surface is entirely due to the electric potential. The distribution of ions in solution in the z-direction, normal to the surface, is then given by the following form of the Boltzmann equation,

$$
N_{i}(z)=N_{i}(\infty) \exp \left(-\frac{z_{i} e \psi(z)}{k T}\right),
$$

where $\mathrm{N}_{\mathrm{i}}(\mathrm{z})$ and $\mathrm{N}_{\mathrm{i}}(\infty)$ are respectively, the number of ions per unit volume of species $i$ at a distance $z$ from the surface, and the number of ions at an infinite distance from the surface and $z_{i}$ denotes the valence of an ion $i$. The charge distribution in the aqueous phase and the electrical potential can be related to each other by the Poisson equation which takes, for this one-dimensional problem, the form

$$
\frac{\mathrm{d}^{2} \psi(\mathrm{z})}{\mathrm{dz}^{2}}=-\frac{\rho(\mathrm{z})}{\varepsilon_{\mathrm{r}} \varepsilon_{0}},
$$


where $\varepsilon_{\mathrm{r}}$ is the uniform dielectric constant of the solution, $\varepsilon_{0}$ the permittivity of free space and $\rho(\mathrm{z})$ denotes the space charge density given by

$$
\rho(\mathrm{z})=\sum_{\mathrm{i}} \mathrm{Z}_{\mathrm{i}} \mathrm{eN} \mathrm{N}_{\mathrm{i}}(\mathrm{z})
$$

A combination of Eqs. 1, 2 and 3 yields the Poisson-Boltzmann equation

$$
\frac{\mathrm{d}^{2} \psi(\mathrm{z})}{\mathrm{dz}^{2}}=-\frac{1}{\varepsilon_{\mathrm{r}} \varepsilon_{0}} \sum_{\mathrm{i}} \mathrm{z}_{\mathrm{i}} \mathrm{eN} \mathrm{N}_{\mathrm{i}}(\infty) \exp \left(-\frac{\mathrm{z}_{\mathrm{i}} \mathrm{e} \psi(\mathrm{z})}{\mathrm{kT}}\right)
$$

The Poisson-Boltzmann equation, Eq. 4, is a nonlinear differential equation which has, in general, to be solved numerically. A first integration of Eq. 4 is analytically possible and results, together with the electroneutrality condition,

$$
\sigma_{\mathrm{m}}=-\int_{0}^{\infty} \rho(\mathrm{z}) \mathrm{dz}
$$

in the Gouy-Chapman equation ${ }^{I}$

$$
\sigma_{\mathrm{m}}=\left[2 \varepsilon_{\mathrm{r}} \varepsilon_{0} \mathrm{kT} \sum_{\mathrm{i}} \mathrm{N}_{\mathrm{i}}(\infty)\left\{\exp \left(-\frac{\mathrm{Zi}_{\mathrm{i}} \mathrm{e} \psi(0)}{\mathrm{kT}}\right)-1\right\}\right]^{\frac{1}{2}}
$$

\footnotetext{
${ }^{1}$ For a more detailed derivation see Appendix A or Aveyard and Haydon (1973).
} 
$\sigma_{\mathrm{m}}$ denotes the surface charge density on the membrane surface and $\psi(0)$ the electric potential at the surface.

The Gouy-Chapman equation shows the relationship between the potential at the surface $(\mathrm{z}=0)$, a quantity of great interest for the adsorption, the surface charge density and the ionic composition. To solve the PoissonBoltzmann equation for any distance $\mathrm{z}$ from the membrane surface a linearization of the Boltzmann factor, instead of a numerical solution, is most often used. The Poisson-Boltzmann equation can be linearized for electric potentials small compared to $\mathrm{kT} / \mathrm{e}=25 \mathrm{mV}$ using the approximation

$$
\exp \left(-\frac{z_{i} \mathrm{e} \psi(\mathrm{z})}{\mathrm{kT}}\right)=1-\frac{\mathrm{z}_{\mathrm{i}} \mathrm{e} \psi(\mathrm{z})}{\mathrm{kT}}
$$

which yields the linearized Poisson-Boltzmann equation

$$
\frac{\mathrm{d}^{2} \psi(\mathrm{z})}{\mathrm{dz}^{2}}=\frac{1}{\varepsilon_{\mathrm{r}} \varepsilon_{0}} \sum_{\mathrm{i}} \mathrm{z}_{\mathrm{i}} \mathrm{eN}_{\mathrm{i}}(\infty) \frac{\mathrm{z}_{\mathrm{i}} \mathrm{e} \psi(\mathrm{z})}{\mathrm{kT}}
$$

The solution of this differential equation is easily obtained as

$$
\psi(\mathrm{z})=\psi(0) \mathrm{e}^{-K z},
$$

where $\mathrm{K}$ is the inverse Debye length,

$$
K=\left(\frac{\mathrm{e}^{2}}{\varepsilon_{\mathrm{r}} \varepsilon_{0} \mathrm{kT}} \sum_{\mathrm{i}} \mathrm{z}_{\mathrm{i}}^{2} \mathrm{~N}_{\mathrm{i}}(\infty)\right)^{\frac{1}{2}}
$$


The electric potential at the surface, $\psi(0)$, can be obtained from the GouyChapman equation, Eq. 6.

The linearized Poisson-Boltzmann equation, Eq. 9, was used in all our calculations and we will refer to the potential obtained in this way as the linearized Gouy-Chapman potential.

\subsection{The Discrete Charge Theory}

Nelson and McQuarrie described, in 1975, an electrostatic model of the membrane-water interface which accounted for discreteness of membrane charge. They considered that the surface charges are discrete rather than uniform and calculated, on this basis, the electric potential distribution in the aqueous phase above the membrane surface. Their discrete charge theory is an extension of the successful Gouy-Chapman theory and differs from the GouyChapman theory in just two points: (1) The membrane surface charge density is modeled as a two-dimensional array of discrete charges instead of a smeared surface charge and (2) the theory allows an embedding of discrete charges below the membrane surface.

The model used for the discrete charge theory is shown in Figure 4. The membrane surface is located at the $\mathrm{z}=0$ plane and an array of charges are located at a distance $c$ behind the membrane surface. The charges in the model of Nelson and McQuarrie are arranged in a periodic manner, on a square lattice. Regions 1 and 2 correspond to the membrane core and membrane surface layer, region 3 to the ionic aqueous solution. Dividing the membrane into two regions 


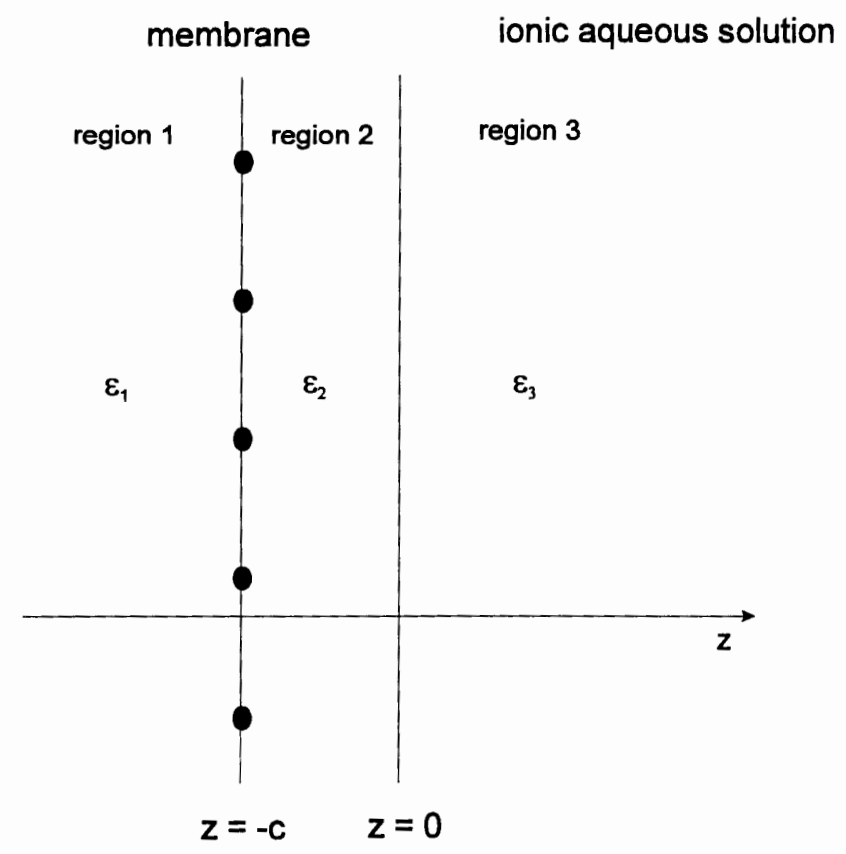

Figure 4. Membrane model used in the discrete charge theory.

takes into account the dielectric inhomogeneity of the membrane. The dielectric constant of the aqueous phase is assumed not to change significantly as a function of distance from the membrane surface.

In the following paragraphs the important elements in the derivation of this model are given. For a more detailed derivation the reader should refer to Nelson and McQuarrie (1975).

The electric potential in region 1 and region 2 satisfy Laplace's equation,

$$
\Delta \psi_{1}=\Delta \psi_{2}=0
$$

In region 3 the electric potential is described by the Poisson equation

$$
\Delta \psi_{3}(\overrightarrow{\mathrm{r}})=-\frac{\rho(\overrightarrow{\mathrm{r}})}{\varepsilon_{3} \varepsilon_{0}}
$$


The electric potentials in all regions are invariant under a lattice transformation as the charges are arranged in a periodic manner and can, therefore, be expanded in a Fourier series of the form

$$
\psi(\overrightarrow{\mathrm{r}})=\sum_{\overrightarrow{\mathrm{G}}} \mathrm{f}_{\overrightarrow{\mathrm{G}}}(\mathrm{z}) \mathrm{e}^{\mathrm{i} \overrightarrow{\mathrm{G}} \overrightarrow{\mathrm{r}}}
$$

where $\mathrm{G}$ is a reciprocal lattice vector, two-dimensional in our case. It is given by

$$
\overrightarrow{\mathrm{G}}=h \overrightarrow{\mathrm{A}}+\mathrm{k} \overrightarrow{\mathrm{B}},
$$

where $\mathrm{h}$ and $\mathrm{k}$ are integers (Miller indices). For the case of a two-dimensional square lattice with lattice constant $a$, the reciprocal lattice vectors are given by

$$
\overrightarrow{\mathrm{A}}=\frac{2 \pi}{\mathrm{a}} \hat{\mathrm{x}} \quad \overrightarrow{\mathrm{B}}=\frac{2 \pi}{\mathrm{a}} \hat{\mathrm{y}}
$$

Substituting Eq. 13 into Eq. 12 the electric potential in region 1 and 2 can be written as

$$
\psi_{1}(\overrightarrow{\mathrm{r}})=\sum_{\overrightarrow{\mathrm{G}}} \mathrm{E}_{\overline{\mathrm{G}}} \mathrm{e}^{\mathrm{i} \overline{\mathrm{G}} \overrightarrow{\mathrm{r}}} \mathrm{e}^{+\mathrm{Gz}}
$$

and

$$
\psi_{2}(\overrightarrow{\mathrm{r}})=\sum_{\overrightarrow{\mathrm{G}}}\left(\mathrm{C}_{\overrightarrow{\mathrm{G}}} \mathrm{e}^{\mathrm{i} \overline{\mathrm{G}} \overrightarrow{\mathrm{r}}} \mathrm{e}^{-\mathrm{Gz}}+\mathrm{D}_{\overrightarrow{\mathrm{G}}} \mathrm{e}^{\mathrm{i} \overrightarrow{\mathrm{G}} \overline{\mathrm{r}}} \mathrm{e}^{+\mathrm{Gz}}\right)
$$


The electric potential in region 3 obeys the Poisson equation, Eq. 13, which together with the space charge density

$$
\rho(\overrightarrow{\mathrm{r}})=\sum_{\mathrm{i}} \mathrm{Z}_{\mathrm{i}} \mathrm{eN} \mathrm{N}_{\mathrm{i}}(\overrightarrow{\mathrm{r}})
$$

and the Boltzmann equation

$$
N_{i}(\vec{r})=N_{i}(\infty) \exp \left(-\frac{Z_{i} e \psi_{3}(\vec{r})}{k T}\right)
$$

results in the following nonlinear differential equation

$$
\begin{aligned}
& \sum_{\overrightarrow{\mathrm{G}}}\left[\frac{\partial^{2}}{\partial \mathrm{z}^{2}} \mathrm{f}_{\mathrm{G}}(\mathrm{z}) \mathrm{e}^{\mathrm{i} \overline{\mathrm{G}} \overrightarrow{\mathrm{T}}}-\left(\mathrm{G}_{\mathrm{x}}{ }^{2}+\mathrm{G}_{\mathrm{y}}{ }^{2}\right) \mathrm{f}_{\overline{\mathrm{G}}}(\mathrm{z}) \mathrm{e}^{\mathrm{i} \overline{\mathrm{G}} \overrightarrow{\mathrm{G}}}\right] \\
& =-\sum_{\mathrm{i}} \frac{\mathrm{Z}_{\mathrm{i}} \mathrm{e} \mathrm{N}_{\mathrm{i}}(\infty)}{\varepsilon_{\mathrm{r}} \varepsilon_{0}} \exp \left(-\frac{\mathrm{Z}_{\mathrm{i}} \mathrm{e}}{\mathrm{kT}} \sum_{\overline{\mathrm{G}}} \mathrm{f}_{\overline{\mathrm{G}}}(\mathrm{z}) \mathrm{e}^{\mathrm{i} \overline{\mathrm{G}} \overline{\mathrm{T}}}\right)
\end{aligned}
$$

This nonlinear differential equation can not be solved analytically. Nelson and McQuarrie assumed in their derivation that the Debye-Hückel theory can be applied to region 3. This is equivalent to the assumption that the Boltzmann factor can be linearized as

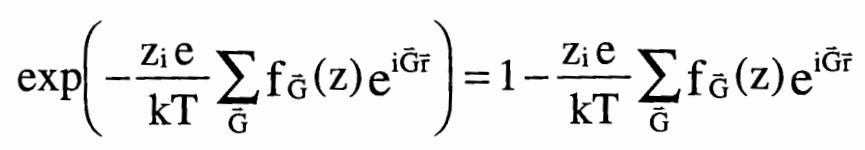

Equation 20 simplifies then into 


$$
\frac{\partial^{2} \mathrm{f}_{\overline{\mathrm{G}}}}{\partial \mathrm{z}^{2}}-\left(\mathrm{G}^{2}+\mathrm{K}^{2}\right) \mathrm{f}_{\tilde{\mathrm{G}}}=0
$$

where $\mathrm{K}$ is the inverse Debye length, already defined in Eq. 10

$$
K=\left(\frac{\mathrm{e}^{2}}{\varepsilon_{\mathrm{r}} \varepsilon_{0} \mathrm{kT}} \sum_{\mathrm{i}} \mathrm{z}_{\mathrm{i}}^{2} \mathrm{~N}_{\mathrm{i}}(\infty)\right)^{\frac{1}{2}}
$$

Using this approximation the electric potential in region 3 can be written as

$$
\psi_{3}(\overrightarrow{\mathrm{r}})=\sum_{\overrightarrow{\mathrm{G}}} \mathrm{A}_{\overline{\mathrm{G}}} \mathrm{e}^{\mathrm{i} \overrightarrow{\mathrm{G}} \overrightarrow{\mathrm{T}}} \mathrm{e}^{-\left(\mathrm{G}^{2}+K^{2}\right)^{\frac{1}{2} \mathrm{Z}}}
$$

The constant $A_{\bar{G}}$ in Eq. 23 can be determined from the boundary conditions,

$$
\begin{gathered}
\psi_{1}(-c)=\psi_{2}(-c) \\
\left.\varepsilon_{1} \frac{\partial \psi_{1}}{\partial z}\right|_{z=-c}=\left.\varepsilon_{2} \frac{\partial \psi_{2}}{\partial z}\right|_{z=-c}+\frac{\sigma}{\varepsilon_{0}} \\
\psi_{2}(0)=\psi_{3}(0) \\
\left.\varepsilon_{2} \frac{\partial \psi_{2}}{\partial z}\right|_{z=0}=\left.\varepsilon_{3} \frac{\partial \psi_{3}}{\partial z}\right|_{z=0}
\end{gathered}
$$


Since the charges are arranged in a periodic manner, the surface charge density $\sigma$ in Eq. 25 can be expanded into a Fourier series

$$
\sigma=\sum_{\overline{\mathrm{G}}} \sigma_{\overline{\mathrm{G}}} \mathrm{e}^{\mathrm{i} \overline{\mathrm{G}} \overline{\mathrm{F}}}
$$

and after some tedious algebra, the following expression for $A_{\bar{G}}$ can be obtained

$$
A_{\overrightarrow{\mathrm{G}}}=\frac{\sigma_{\overrightarrow{\mathrm{G}}}}{\varepsilon_{0}} .
$$

$\overline{\varepsilon_{1} G \cosh G c+\left(\varepsilon_{3} \varepsilon_{1} / \varepsilon_{2}\right)\left(G^{2}+K^{2}\right)^{\frac{1}{2}} \sinh G c+\varepsilon_{2} G \sinh G c+\varepsilon_{3}\left(G^{2}+K^{2}\right)^{\frac{1}{2}} \cosh G c}$

The factor $\sigma_{\overline{\mathrm{G}}}$ for a simple square lattice with one ion of charge $q$ located at each lattice point, is given by ${ }^{2}$

$$
\sigma_{\overrightarrow{\mathrm{G}}}=\mathrm{q} / \mathrm{a}^{2}
$$

Finally, a combination of Eqs. 23, 29 and 30 and the simplifying assumption that the membrane region has a uniform dielectric constant, $\varepsilon_{1}=\varepsilon_{2}$, results in

$$
\begin{array}{r}
\psi_{3}(\overrightarrow{\mathrm{r}})=\frac{\mathrm{q}}{2 \pi \mathrm{a} \varepsilon_{0}} \sum_{\mathrm{h}, \mathrm{k}} \frac{1}{\exp \left(\sqrt{\mathrm{h}^{2}+\mathrm{k}^{2}} \cdot 2 \pi \mathrm{c} / \mathrm{a}\right)} \cdot \frac{1}{\varepsilon_{1}\left(\mathrm{~h}^{2}+\mathrm{k}^{2}\right)+\varepsilon_{3} \sqrt{\mathrm{h}^{2}+\mathrm{k}^{2}+\mathrm{m}^{2}}} \\
\cos (2 \pi \mathrm{h} \mathrm{x} / \mathrm{a}+2 \pi \mathrm{ky} / \mathrm{a}) \cdot \exp \left(-\sqrt{\mathrm{h}^{2}+\mathrm{k}^{2}+\mathrm{m}^{2}} \cdot 2 \pi \mathrm{z} / \mathrm{a}\right)
\end{array}
$$

where $\mathrm{m}=\mathrm{Ka} / 2 \pi$.

\footnotetext{
${ }^{2}$ For a detailed derivation see Nelson and McQuarrie (1975).
} 
This expression, Eq. 31, was used in all our calculations. Below we refer to this electric potential as the discrete charge potential.

\subsection{The Boltzmann Factor}

The Boltzmann factor, $\mathrm{Bf}_{\mathrm{z}_{\mathrm{i}}}(\overrightarrow{\mathrm{r}})$, for an ion of valence $\mathrm{z}_{\mathrm{i}}$ is defined as

$$
\mathrm{Bf}_{\mathrm{z}_{\mathrm{i}}}(\overrightarrow{\mathrm{r}})=\exp (-\mathrm{ez} \psi(\overrightarrow{\mathrm{r}}) / \mathrm{kT})
$$

The Boltzmann factor relates bulk and interfacial concentrations of ions and plays an important role in ion adsorption to biological membranes. 


\section{Electrical Potential and Ion Concentration Distribution in the Vicinity of the Charged Membrane Surface}

\subsection{Computations}

The linearized Gouy-Chapman potential, Eq. 9, the discrete charge potential, Eq. 31, and the respective Boltzmann factors were calculated using a Visual Basic computer program. The calculations were done on IBM compatible $486 / 33$ computer.

The important elements of this program are:

(1) Determination of the ionic composition of the aqueous solution and the calculation of the Debye length.

(2) Determination of the Gouy-Chapman potential at the membrane surface from Eq. 6 for given surface charge density and ionic composition of aqueous solution.

(3) Calculation of the linearized Gouy-Chapman potential according to Eq. 9. 
(4) Calculation of the discrete charge potential according to Eq. 31.

(5) Calculation of the Boltzmann factor, Eq. 32, for hexavalent positively charged Ruthenium Red ions for two cases: (i) the discrete charge model and (ii) the smeared charge electrostatic model.

The computations in steps (1) to (3) and (5) are straightforward. By performing steps (1) to (3), the linearized Gouy-Chapman potential was obtained for given experimental conditions such as surface charge density and ionic composition of the aqueous solution. Boltzmann factors in step (5) were calculated according to Eq. 32. The calculations of the discrete charge potential were performed by summations over $\mathrm{h}$ and $\mathrm{k}$ from -49 to +49 . This corresponds to a square array of $99 \times 99$ discrete charges on the membrane surface. The relatively large array was necessary to avoid oscillations in the potential and to obtain correct results at distances close to the membrane surface. ${ }^{3}$ We have taken the advantage of the symmetry of the square lattice so that it was necessary to calculate potentials only for a quarter of the square unit cell $(0 \leq x \leq a / 2$ and $0 \leq y \leq a / 2)$. The potential in this quarter cell was obtained for 100 equidistant grid points to achieve a good spacial resolution of the potential and to make possible accurate averaging of both the potential and the Boltzmann factor. Potential profiles for the whole array or an arbitrary section of the array can then be easily obtained

\footnotetext{
${ }^{3}$ Oscillations in the potential occur due to the Fourier series expansion of the potential. The accuracy of the expansion increases with the size of the array and high orders of Fourier series expansion are particularly important for calculations of the potentials close to the membrane surface since the potential rapidly changes there.
} 
by shifting and mirroring this quarter cell. This approach speeded considerably the computations (on a scale of minutes).

\subsection{Results}

In all the calculations we assumed a dielectric constant for the aqueous phase of $\varepsilon_{3}=78.5$ and considered the membrane as a homogenous region with a dielectric constant, $\varepsilon_{1}=\varepsilon_{2}=3$. The ionic composition was $0.03 \mathrm{M}$ monovalent salt, citrate/borate/phosphate $(2 \mathrm{mM} / 2 \mathrm{mM} / 0.5 \mathrm{mM})$ buffer, $1 \mathrm{mM}$ Ruthenium Red, $\mathrm{pH} 7$, unless stated differently.

Figure 5 and Figure 6 show spacial distributions of the electric potential and ion concentration potential profiles for the discrete charge model. These profiles were calculated for three different distances, $\mathrm{z}$, from the membrane surface, $z=1 \AA, z=2 \AA$ and $z=5 \AA$. Due to the screening effect of the counterions, the magnitude of the potential decreases very rapidly with the distance from the membrane surface. At the membrane surface, the potential distribution looks like a set of delta functions. Due to the effective screening only very small humps in the potential profile can be seen at a distance $5 \AA$ from the membrane surface.

Concentration profiles, as can be seen in Figure 6 are very different. The extreme sharpness of the ion concentration peaks is because the ion concentration depends exponentially on the electric potential (recall the Boltzmann factor). The computed results illustrate the potentially important role of membrane discrete charges on the ion distribution in the vicinity of the membrane surface. 
The dependence of the Gouy-Chapman and the discrete charge potential on the distance $\mathrm{z}$ from the membrane surface is illustrated in Figure 7 . In this figure we compare electric potentials at several locations within the square unit cell of a charge array. The "minimum" potential corresponds to the center of the square unit cell whereas the "maximum" potential to the region above the discrete charge. The solid curves depict the "average" potential obtained by averaging the electric potential distribution over the unit cell. The ionic composition in Figure 7(a) and (b) differs only by the absence and the presence of Ruthenium Red (1mM). As can be seen in Figure 7(a) the "average" potential obtained from the discrete charge model and the Gouy-Chapman potential obtained from the smeared charge model are nearly identical. The breakdown of the Nelson and McQuarrie model is seen in Figure 7(b) which shows a discrepancy between the "average" and the Gouy-Chapman potential in the presence of a multiply charged counterion. This is because of the inapplicability of the linearization of the Boltzmann factor (see Eq. 21).

Figure 8 shows that "minimum" (Figure 8b) and "maximum" (Figure 8a) potentials converge with increasing embedding depth of the membrane charges to the "average" potential. The average potential did not change with embedding depth. In addition, the embedding of membrane charges causes smoothing of spacial variation of electric potential. The differences between electric potential computed for the discrete charge and the smeared charge distribution progressively decrease with the increase of the embedding depth.

A plot of the electric potential as a function of surface charge density is given in Figure 9 (the potential is calculated at a distance $z=0.1 \mathrm{~nm}$ from the membrane surface and for charges at the membrane surface). This plot shows that the discrepancy between the smeared charge and discrete charge model 
increases with increasing surface charge density: the "average" negative potential increases rapidly with the increasing surface charge density in a linear fashion. In contrast the increase in the Gouy-Chapman potential with the surface charge density is less rapid.

The Boltzmann factors as a function of distance $\mathrm{z}$ from the membrane surface are shown in Figure 10. The differences between the Boltzmann factors obtained for the discrete charge model and the Gouy-Chapman model are enormous but their occurrence is probably to a great extent caused by the invalid approximation of linearization of the Boltzmann factor, Eq. 21, done in the discrete charge model. Evidence of the inconsistency of the linearization of the Boltzmann factor can be seen when comparing the plot of the linearized Boltzmann factor, designated as "linearized discrete charge model", with the non-linearized Boltzmann factor obtained with the discrete charge model. If the linearization of the Boltzmann factor according to Eq. 21 is valid, the Boltzmann factor obtained for the discrete charge potential should be approximately the same as that for the linearized and the non-linearized Boltzmann factor. The great gap between the non-linearized and the linearized Boltzmann factor shows the breakdown of the linearization of the Boltzmann factor in the discrete charge theory.

The embedding of the charges in the membrane causes a decrease in the Boltzmann factor obtained for the discrete charge theory, as can be seen in Figure 11(a) and (b) whereas the embedding cannot be calculated with the current version of the Gouy-Chapman theory. The gap between Boltzmann factors increases with the increasing surface charge density very rapidly. According to Voelker (1994) the Boltzmann factor obtained in experiments with hexavalent Ruthenium Red was found to be smaller than the Boltzmann 
factor obtained from the Gouy-Chapman theory. This discrepancy indicates the inapplicability of the current version of the discrete charge theory. We conclude that the discrete charge theory cannot be used as a tool to predict the effect of discrete charges, the reason is that the approximation on which the current theory has been constructed for nearly all real experimental conditions breaks down. 

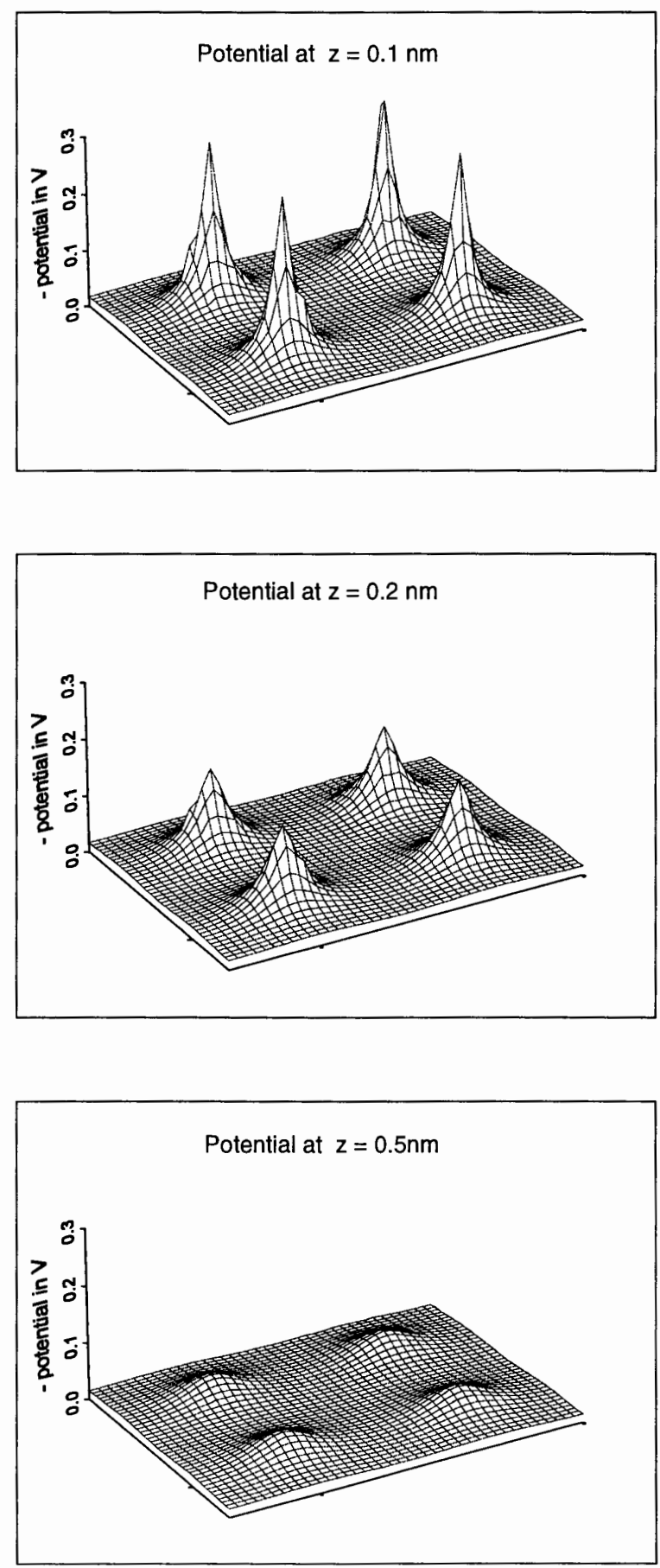

Figure 5. Potential profiles for various distances $\mathrm{z}$ from the membrane surface; the charges are located directly at the membrane surface $(\mathrm{c}=0)$, surface charge density $=0.126 \mathrm{e} / \mathrm{nm}^{2}$ (corresponds to a lattice constant $\mathrm{a}=2.8 \mathrm{~nm}$ ), and Ruthenium concentration $=1 \mathrm{mM}$. 

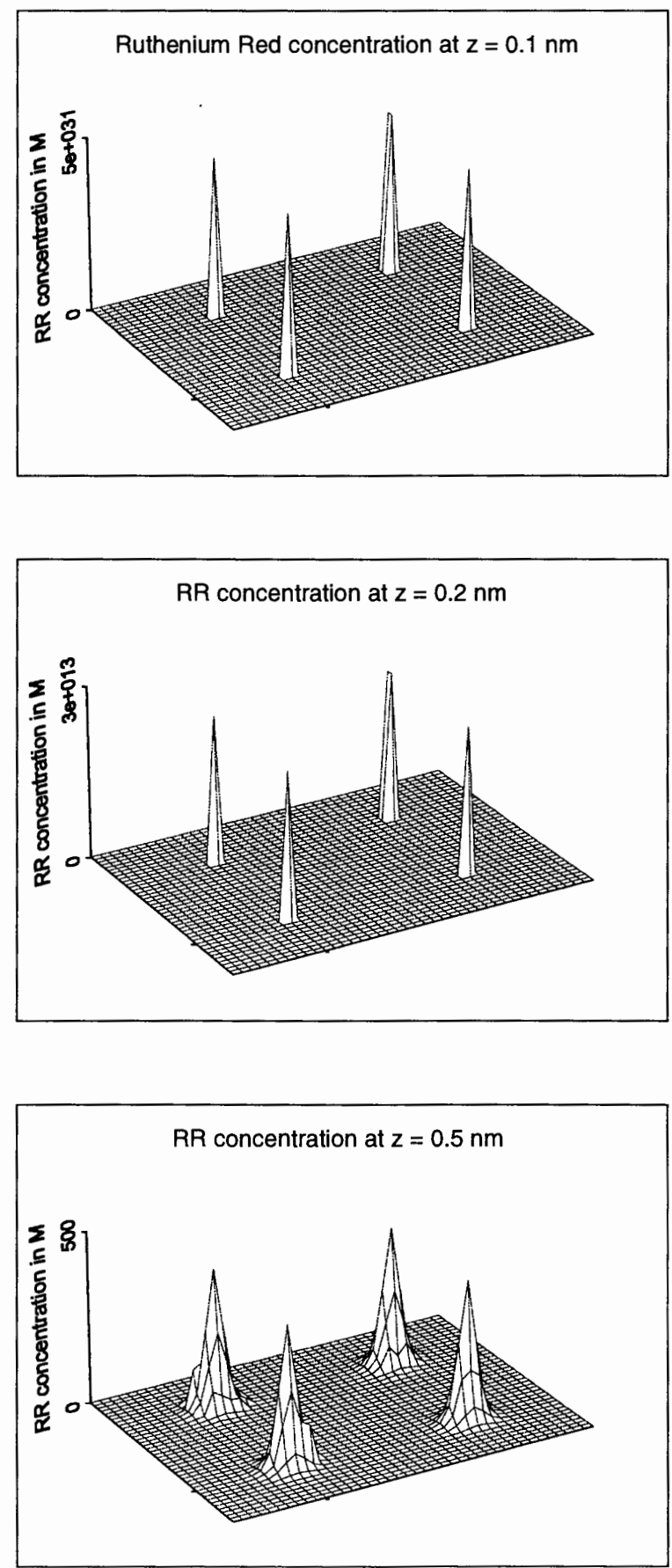

Figure 6. Concentration profiles for various distances $\mathrm{z}$ from the membrane surface; parameters are the same as in Figure 5. 

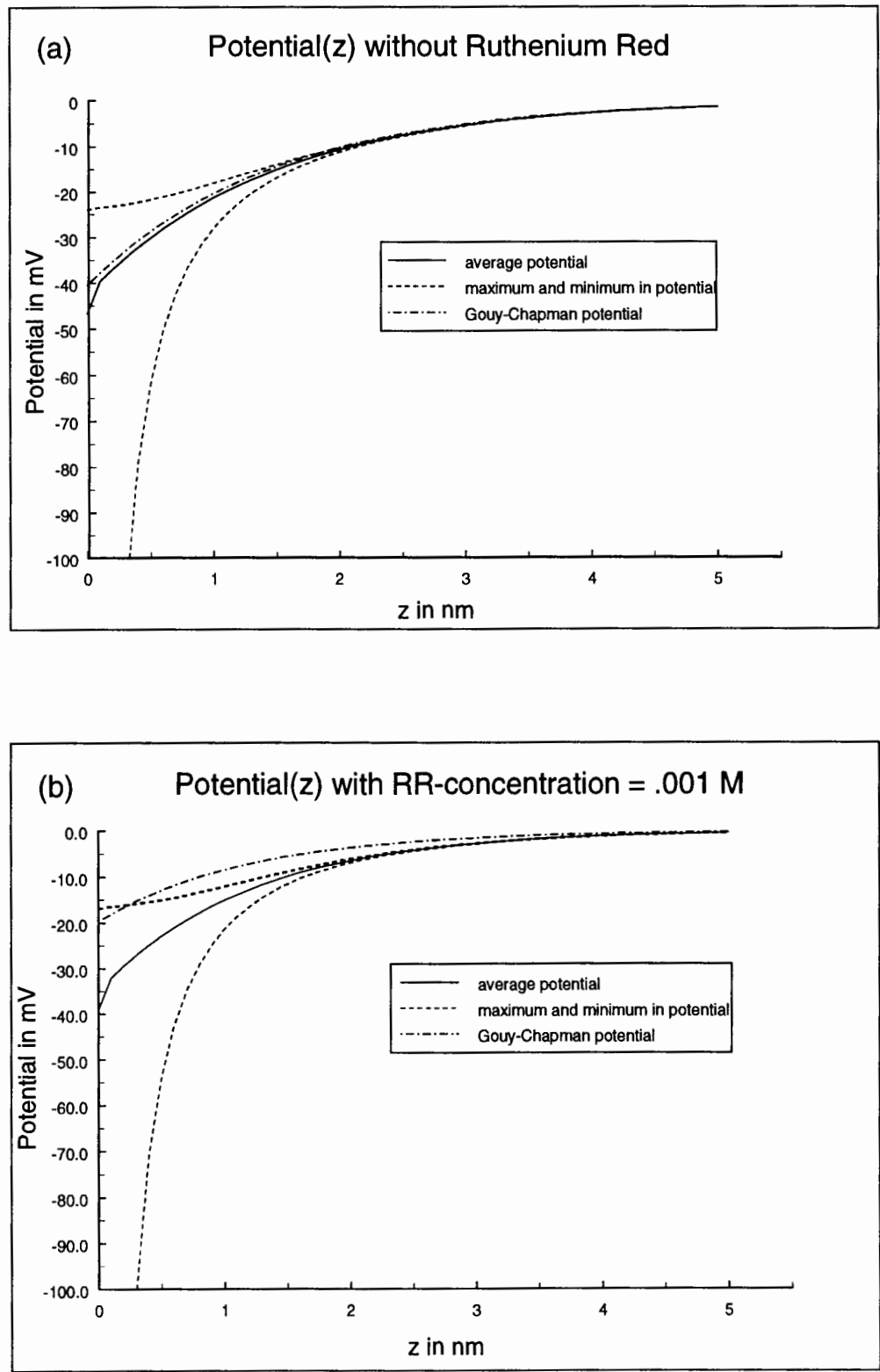

Figure 7. The potential as a function of distance from the membrane surface; the charges are directly located at the membrane surface $(c=0)$, and the surface charge density is $0.126 \mathrm{e} / \mathrm{nm}^{2}$ (corresponds to a lattice constant $\mathrm{a}=2.8 \mathrm{~nm}$ ). The graph (a) shows the potential without Ruthenium Red in the aqueous solution, the graph (b) the potential with a $1 \mathrm{mM}$ Ruthenium Red concentration. 

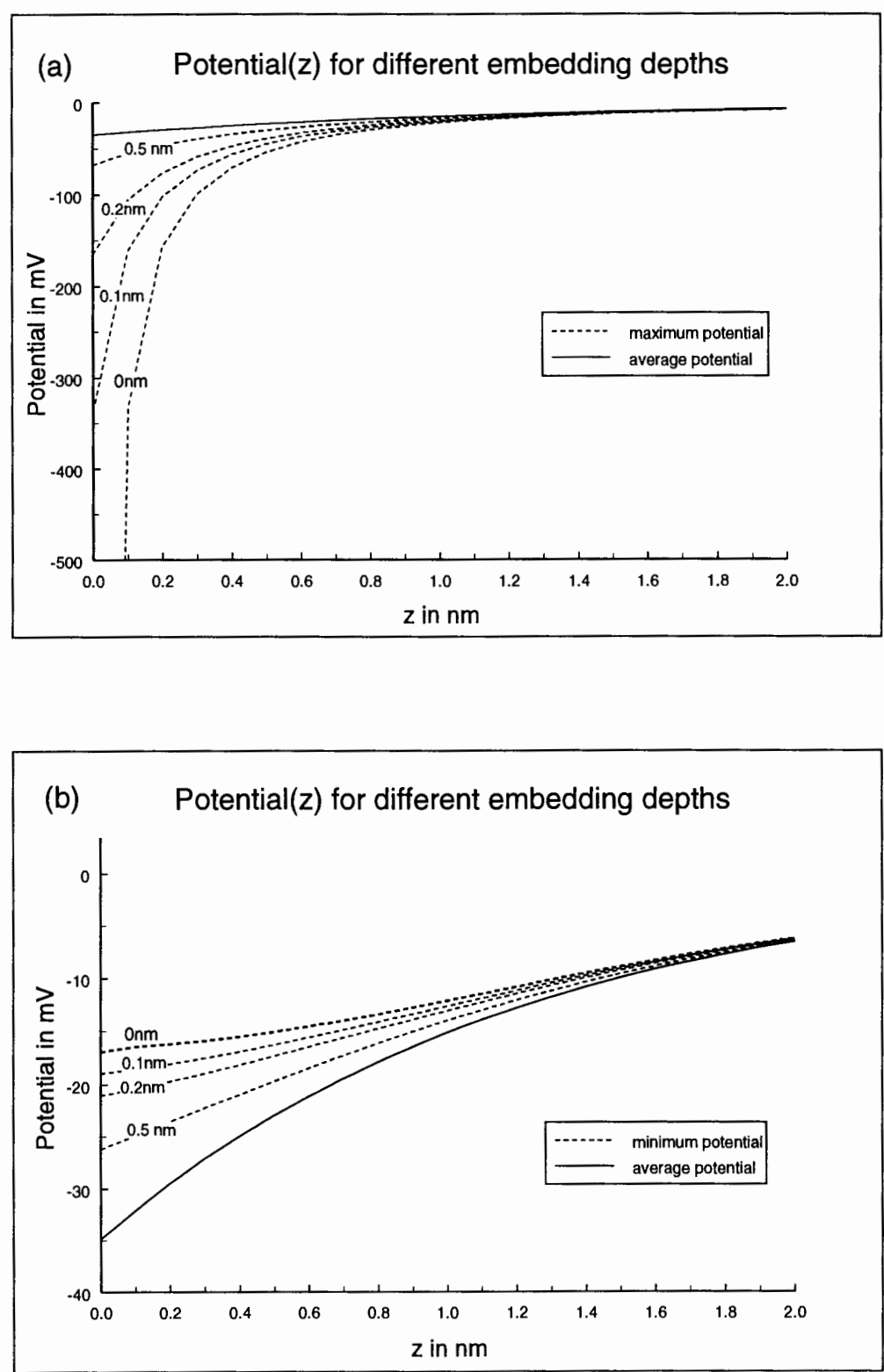

Figure 8. The potential as a function of the distance $\mathrm{z}$ from the membrane; for surface charge density $0.126 \mathrm{e} / \mathrm{nm}^{2}$. The potentials, (a) maximum potential, (b) minimum potential, are calculated for the embedding depths $\mathrm{c}=0 \mathrm{~nm}, \mathrm{c}=0.1$ $\mathrm{nm}, \mathrm{c}=0.2 \mathrm{~nm}$ and $\mathrm{c}=0.5 \mathrm{~nm}$. 


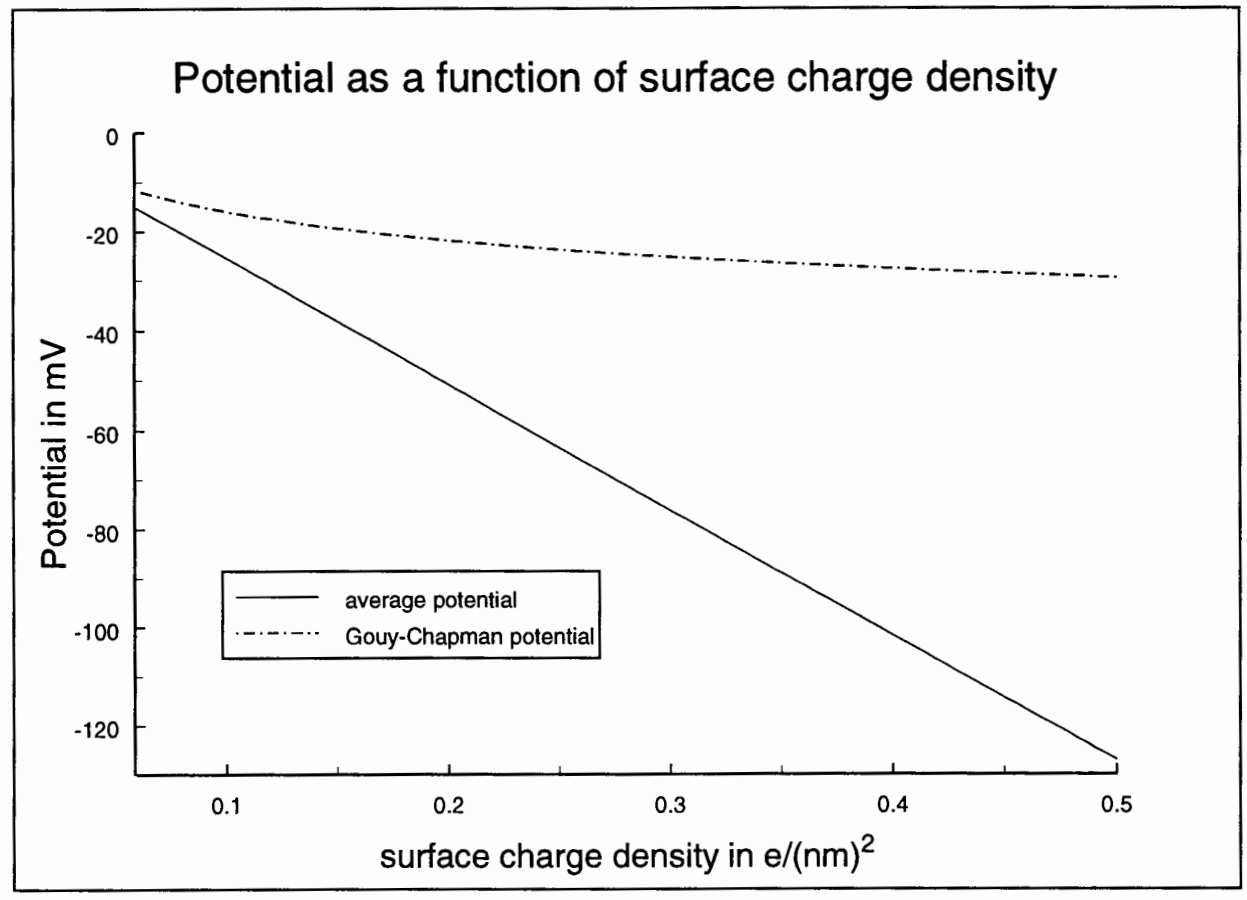

Figure 9. The electric potential as a function of surface charge density. Charges are assumed to be located directly at the membrane surface $(c=0)$, the potential was calculated for distance $\mathrm{z}=0.1 \mathrm{~nm}$ from the membrane surface. 


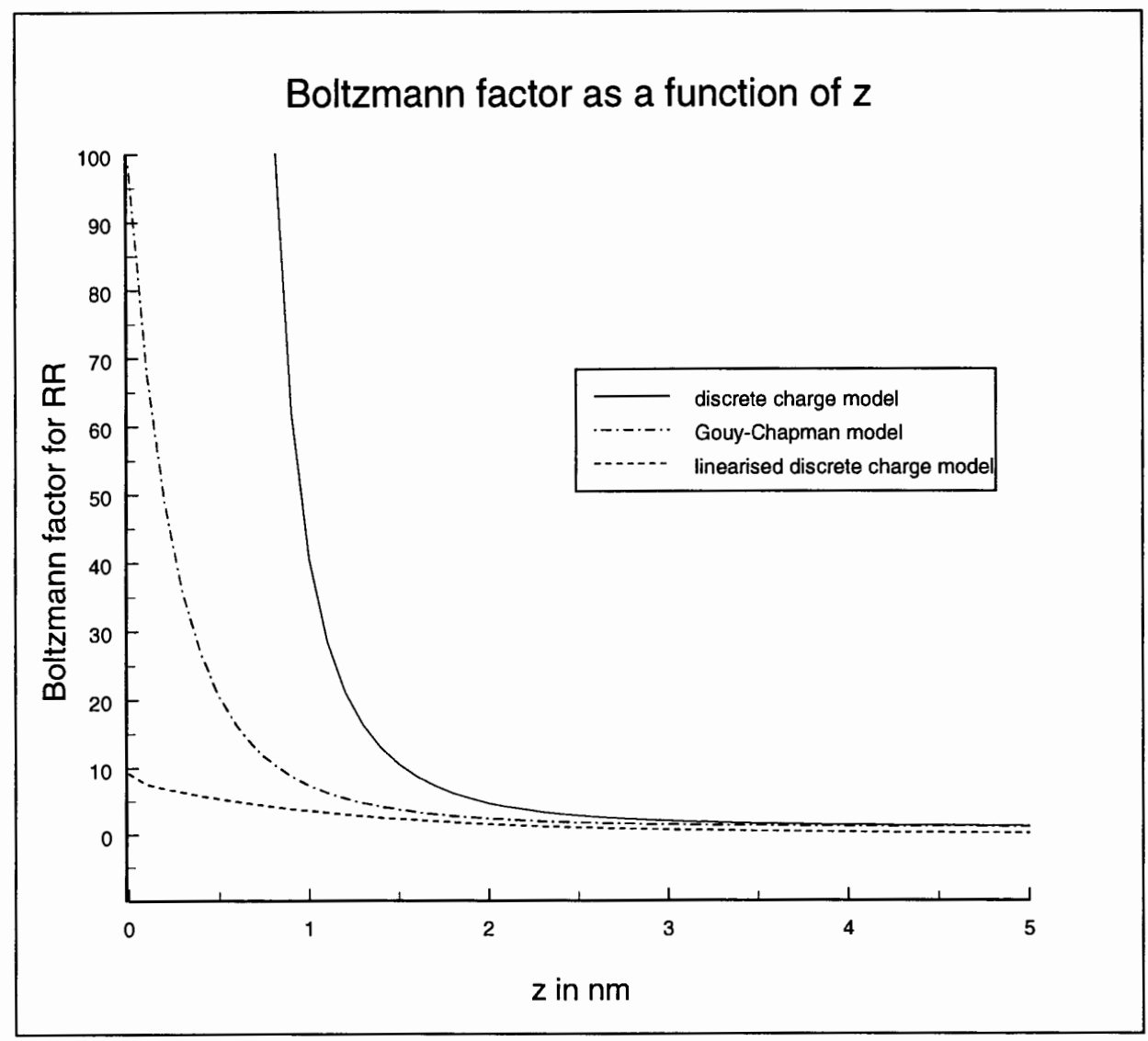

Figure 10. The Boltzmann factor as a function of distance $\mathrm{z}$ from the membrane surface. Charges are assumed to be located directly at the membrane surface ( $\mathrm{c}=$ 0 ), the surface charge density $0.126 \mathrm{e} / \mathrm{nm}^{2}$. 

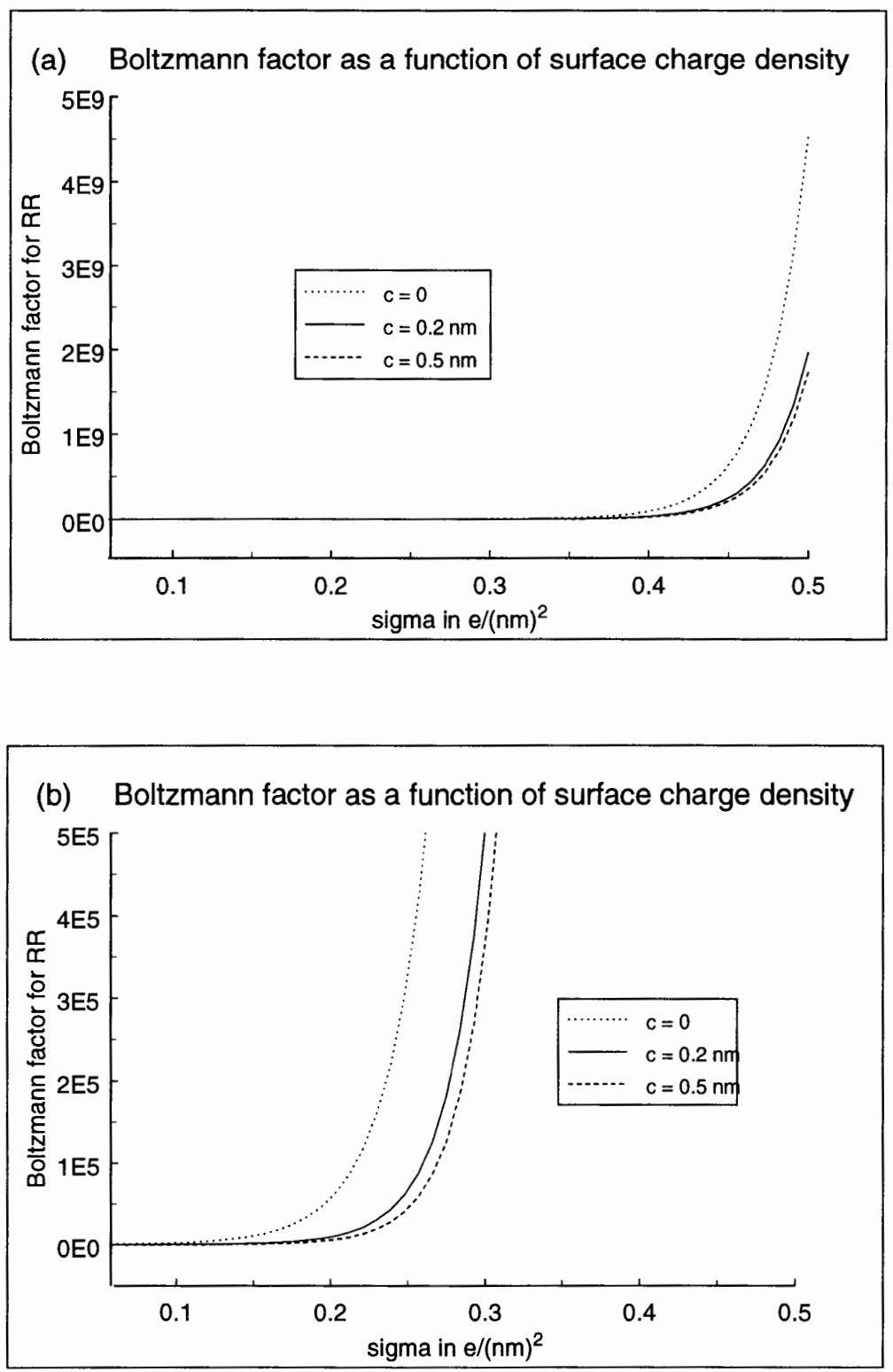

Figure 11. The dependence of Boltzmann factor on the surface charge density; (a) the Boltzmann factor for the discrete charge model, (b) an enlarged part of (a). The Boltzmann factor was calculated at adistance $\mathrm{z}=5 \AA$ from the membrane surface. 


\section{Part II}

\section{Distribution of 2,4,6-Trichlorophenol in a Membrane Model System}




\section{Introduction}

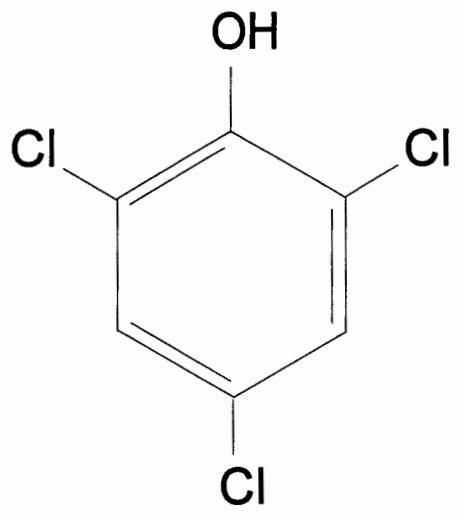

Figure 12.

Molecular structure of 2,4,6-Trichlorophenol
In this research project we determine the distribution of 2,4,6-Trichlorophenol $(2,4,6-$ $\operatorname{TrCP}$ ) in the octanol-water system which is used as a model system for biological membranes. The octanol-water system - a detailed description of which is given in section 4.1.2 - is widely used for modeling adsorption and partitioning of hydrophobic compounds, such as 2,4,6-TrCP.

The chemical structure of 2,4,6-TrCP is

shown in Figure 12. Chlorophenols exist either in an un-ionized or an ionized form depending on the acidity of the aqueous environment. The dissociation constants of chlorophenols are typically $10^{-3}-10^{-9}$ which corresponds to $\mathrm{pKa}$ values of 3-9. The pKa value of 2,4,6-TrCP is 6.1 (Schellenberg et al., 1984).

The distribution of un-ionized species of chlorophenols in the octanolwater system has been studied earlier (Schellenberg et al., 1984; Callahan et al., 1979; Xie et al., 1984 and Leo et al., 1971), however data for the distribution of the ionized species are not available. Furthermore, the suitability of the octanolwater system as a model for biomembranes has not been demonstrated for the ionized species. In the present work we report results on the distribution of 2,4,6-TrCP for both ionized and un-ionized species and compare our results with those obtained from adsorption studies done on lipid membranes under the same experimental conditions. 
The study and the development of membrane adsorption models for 2,4,6-Trichlorophenol as well as for other chlorophenols is of great interest as the chlorophenols are strong biocides and are present in the environment due to their wide industrial and agricultural use. Their relatively high toxicity raises questions concerning the environmental impact of these materials. The behavior in which toxic compounds move and are distributed in the environment is complex. This project contributes to the development of understanding of distribution of $2,4,6-\operatorname{TrCP}$ in the environment by obtaining data on the distribution of this compound in a model, the octanol-water system.

Below we present information on the occurrence of chlorophenols in the environment and on their toxicity.

The most widely used chlorophenol was pentachlorophenol (PCP). This chemical was extensively used as an industrial biocide and as an agricultural pesticide (Beynon et al., 1981).

Tetrachlorophenols (TeCP's) and trichlorophenols (TrCP's) are also found in the environment, often as degradation products of PCP. The compound of our present interest, 2,4,6-TrCP, has been used in the past as a germicide, bactericide, wood and glue preservative, and as an antimildew agent (Sittig, 1985). Various TeCP's and other TrCP's, including 2,4,6-TrCP are byproducts of chlorobleaching of pulp and therefore present in the wastewater from wood pulp bleaching mills (Paasivirta et al., 1992). Breakdown processes of PCP through sunlight (Mikesell and Boyd, 1986) and anaerobic biodegredation (Nicholson et al., 1992) are major sources of all the different chlorinated phenols even if they are not directly used in industry or agriculture.

The production of PCP and therefore the introduction of all chlorophenols into the environment started in the 1930's and reached by 1981 a 
worldwide production of 50000 metric tones, as estimated by Beynon and coworkers (Beynon et al., 1981). The mass production and extensive use of PCP has been discontinued and is today restricted in many countries such as Sweden, USA, Japan and Germany. Even though the use of PCP has been restricted nowadays, the extensive and wide use in the past has had an enormous impact on environment and chlorophenols are still major pollutants.

Wild et al. (1992) estimated that about $90 \%$ of the environmental burden of PCP is associated with the soil, water follows as the next significant sink. Humans are also exposed to PCP and other chlorophenols. The primary exposure route of chlorophenols is most likely via ingestion of contaminated food and water, inhalation of vaporized chlorophenols and skin absorption by direct contact with laundry products, wooden goods, leather, paper and textiles. The main route of PCP uptake by the general population is by ingestion of contaminated food, whereas uptake due to inhalation of vaporized PCP plays an important role for occupationally exposed individuals. Geyer et al. (1987) reported a daily PCP ingestion of an average adult to be about $19 \mu \mathrm{g}$. The PCP body burden of humans ranges, according to literature values, between $533 \mu \mathrm{g}$ and $673 \mu \mathrm{g}$ and PCP is mainly concentrated in the liver and the brain (Geyer et al., 1987; Hattemeyer-Frey and Travis, 1989; and Wild and Jones; 1992). In another study by Mussalo-Rauhamaa et al. (1989) the concentrations of 2,4,6TrCP, 2,3,4,6-TeCP and PCP in the tissues of people from Finland were studied. The $\operatorname{TrCP}$ and $\mathrm{TeCP}$ concentrations were found to be significantly lower than PCP concentrations. 2,4,6-TrCP was not detectable. The presence of $2,4,6-\operatorname{TrCP}$ at a level of $12 \mu \mathrm{g} / \mathrm{l}$ indicates an additional lifetime cancer risk of 1 in 100,000 (Sittig, 1985). 
Studies of human exposure to chlorophenols can give only a rough idea of the factors and consequences involved in potential human exposure. Knowledge of the presence and fate of chlorophenols in sediments, soil, air, water, and organisms is therefore essential to relate their persistence and movement in the environment to potential human exposure. It is not possible to evaluate all polluted sites and to account for all conditions associated with the presence of chlorophenols in the environment. Therefore models, such as the octanol-water system, that enable us to predict chlorophenol concentrations under various conditions are essential.

On the cellular level, chlorophenols are found to be toxic due to their action as uncouplers of oxidative phosphorylation. ${ }^{4}$ ATP synthesis is strongly coupled to the $\mathrm{H}^{+}$-gradient across the inner mitochondrial membrane. A lowering of this $\mathrm{H}^{+}$-gradient due to the activity of chlorophenols, as they transport protons across the membrane, disrupts the coupling between this $\mathrm{H}^{+}$gradient and the ATP synthesis. If the uncoupling effect is strong, not enough ATP can be produced to supply the energy for all cell functions and the cell will die.

The ability of chlorophenols to act as carriers of $\mathrm{H}^{+}$-ions can be described by two different mechanisms: A neutral chlorophenol (HA) permeates the membrane and releases a $\mathrm{H}^{+}$-ion at the more basic matrix side of the membrane, the ionized chlorophenol $\left(\mathrm{A}^{-}\right)$diffuses back to the more acidic side, picks up another $\mathrm{H}^{+}$-ion to release it again on the more basic side (Terada 1990). Another alternative to the back diffusion is the transmembrane flow of AHA dimers (Smejtek et al., 1976). Both mechanisms show that the uncoupling activity depends strongly on the lipophilicity of the chlorophenol,

\footnotetext{
${ }^{4} 2,4,6-\operatorname{TrCP}$ is also a carcinogen (Sittig, 1985).
} 
the greater the adsorption and the greater the rate of permeation through the membrane the stronger is the uncoupling effect. The weak acidity of chlorophenols, their ability to exist as phenolate ions, is necessary to make the transfer of protons possible.

Adsorption studies of chlorophenols to biological membranes serve two different purposes: (1) Adsorption studies of chlorophenols to biological membranes provide a better understanding of their toxicity and (2) adsorption to biological membranes plays an important role in estimating the concentration of the chlorophenols in soils, sediments and organisms as all these materials contain membranes and membrane fragments. ${ }^{5}$

The adsorption or partitioning of the chlorophenols from water into biological cells or organic components of sediments and soils (Schellenberg et al. 1984, Lagas 1988) has been extensively modeled by the transfer of these chemicals from water to octanol. We use therefore, the octanol-water system as a model system in our experiments. For the description of the partitioning in the octanol-water system, the octanol-water partition coefficient is defined by

$$
P_{o w}=\frac{[X]_{o}}{[X]_{w}}
$$

where $[\mathrm{X}]_{\mathrm{o}}$ is the concentration of the compound of interest in octanol and $[\mathrm{X}]_{\mathrm{w}}$ is its concentration in water. The octanol-water partition coefficient is a widely used tool and has numerous applications in addition to predicting the presence of toxic compounds in the environment.

\footnotetext{
${ }^{5}$ Soil and sediments contain biological membranes due to the presence of live and dead cells and cell fragments (Wang, Goving and Dobbs 1993).
} 
The octanol-water partition coefficient can be obtained by a direct measurement and to a certain degree by calculation from known physicochemical properties of a chemical. Approaches to obtain partition coefficient are many, some of them are combining experimental measurements and quantum-mechanical calculations. $^{6}$

In our research we used the shake flask method: the examined compound $\mathrm{X}$ was injected into a two-phase system consisting of known volumes of octanol and water. After shaking the solution to reach the equilibrium distribution, the two phases were separated and the concentrations in each phase determined. This procedure belongs to a class of direct methods of measurements of $\mathrm{P}_{\text {ow }}$. A more detailed description of this method is given in chapter 6 .

The usefulness of octanol-water partition coefficients rests on the assumed similarity of the energetics of transfer of hydrophobic chemicals from water into octanol to that from water into biological membranes. We question this similarity for ionized chlorophenols since a significant discrepancy between the partitioning in the octanol-water system and adsorption to phospholipid vesicles was found for PCP. Smejtek and Wang (1993) compared partitioning of PCP in the octanol-water system with the adsorption to phospholipid vesicles and found substantial differences for $\mathrm{pH}>\mathrm{pKa}$. The energetics of transfer of

\footnotetext{
${ }^{6}$ The different ways of determining octanol-water partition coefficients include several different experimental methods and theoretical approaches which use the physico-chemical properties of the partitioning species to predict the octanol-water partition coefficient. Other methods use: reversedphase high-performance liquid chromatography (RP-HPLC) (Klein et al., 1988), calculation of $\mathrm{P}_{\mathrm{OW}}$ from water solubility (Miller et al., 1985), from chemical structure which include factors like shape, molecular volume, total molecular surface area (TSA) and total molecular volume (TMV) of a compound (Bruijin and Hermens 1990, Sabijic et al., 1993). A critical review of the measurements of octanol-water partition coefficients is given by Chessells et al. (1991).
} 
the ionized species of PCP in octanol-water and membrane-water system was found to be not comparable: the octanol-water partition coefficient of the ionized species of PCP was several orders of magnitude smaller than the membrane-water partition coefficient.

The goal of our study was to measure the octanol-water partition coefficient of $2,4,6-\operatorname{TrCP}$ as a function of $\mathrm{pH}$ in order to obtain partition coefficients for the un-ionized as well as ionized species of 2,4,6-TrCP and to compare the obtained data with results of Blochel (1992) who measured adsorption of ionized chlorophenols to lipid membranes under the same conditions. The objective was to test the applicability of the octanol-water partition coefficient for the phenolate of 2,4,6-TrCP to membrane adsorption. 


\section{Theory}

\subsection{Partition of Un-ionized and Ionized Molecules Between Water and a Medium of Low Polarity}

The distribution of 2,4,6-TrCP between octanol and an aqueous phase as a function of $\mathrm{pH}$ was measured. It is important to recognize that the partitioning of 2,4,6-TrCP between octanol and water is not an ideal partitioning process ${ }^{7}$. Octanol and water are partially miscible and therefore the two phases are not ideal. In addition, 2,4,6-TrCP exists in the aqueous phase in two forms: ionized and un-ionized. The ionization is $\mathrm{pH}$ dependent and each species is exhibiting totally different partitioning behavior. Buffer components, which are expected to be strongly coupled to the partitioning of the ionized species, also contribute to the non-ideal behavior of the system.

We present first the concepts of ideal partitioning (Gerthsen et al., 1986; Herrmann, 1993) since they provide the foundation for the understanding of distribution of ionized and un-ionized species between octanol and aqueous phase. A short overview of thermodynamics given in section 4.1 .1 provides the necessary insight into the basic properties of an ideal partitioning system. In

\footnotetext{
${ }^{7}$ Ideal partitioning assumes two homogenous extended bulk phases, ideal gas behavior of the solutes and independent partitioning of each species in case of the presence of several compounds.
} 
4.1.2 we describe the octanol-water system. The definitions of the various partition parameters for membrane-water system follow in 4.1.3.

\subsubsection{Ideal Partitioning}

The distribution of several substances between two phases takes place in such a way so that the entropy of the system is maximized. Using the fact that pressure and temperature are constant during the partitioning process, the maximization of the entropy is equivalent to minimization of the free energy, G. The free energy $G$ is defined by

$$
G(T, p, n)=U+p V-T S
$$

where $\mathrm{T}$ is the temperature, $\mathrm{p}$ the pressure, $\mathrm{n}$ the number of particles, $\mathrm{U}$ the internal energy and $S$ the entropy.

First we consider a system with just one solute. It is assumed that each species behaves like an ideal gas and its behavior is independent of the concentration of the other compounds present in the system. This concept can be easily generalized to a system with several components.

The differential of the free energy is given by

$$
d G=\frac{\partial G(T, p, n)}{\partial T} d T+\frac{\partial G(T, p, n)}{\partial p} d p+\frac{\partial G(T, p, n)}{\partial n} d n
$$

From the definition of free energy follows that 


$$
\begin{aligned}
d G & =d(U+p V-T S) \\
& =T d S-p d V+\mu d n+p d V+V d p-T d S-S d T \\
& =V d p-S d T+\mu d n
\end{aligned}
$$

At constant temperature and pressure the fist two terms in Eq. 34 are zero since $\mathrm{dT}=0$ and $\mathrm{dp}=0$. Then the change of free energy is given by

$$
d G=\mu d n
$$

From the comparison of Eqs. 34 and 35 follows the definition of the chemical potential, $\mu$.

$$
\mu(T, p, n)=\frac{\partial G(T, p, n)}{\partial n}
$$

Using one of Maxwell's relations

$$
\frac{\partial^{2} G(T, p, n)}{\partial p \partial n}=\frac{\partial V(T, p, n)}{\partial n}=\frac{\partial \mu(T, p, n)}{\partial p}
$$

and applying the ideal gas law

$$
p V=n R T
$$

we obtain a differential equation relating changes in chemical potential to changes in pressure. 


$$
d \mu(T, p, n)=\frac{R T}{p} d p
$$

Integration of Eq. 40 , assuming that the standard chemical potential, $\mu_{0}^{*}$, corresponds to the pressure, $\mathrm{p}_{0}$, yields,

$$
\mu=\mu_{0}^{*}+\mathrm{RT} \ln \frac{\mathrm{p}}{\mathrm{p}_{0}}
$$

This relationship between the pressure and the chemical potential is not very useful for the description of transfer of molecules between two dense media. However the pressure can be easily related to the molar concentration, $c$, of a partitioning compound by applying the ideal gas law, Eq. 39,

$$
p=\frac{n}{V} R T=c R T
$$

This results in

$$
\mu=\mu_{0}^{*}+\mathrm{RT} \ln \left(\frac{\mathrm{c}}{\mathrm{c}_{0}}\right)=\mu_{0}^{*}-\mathrm{RT} \ln \mathrm{c}_{0}+\mathrm{RT} \ln \mathrm{c}=\mu_{0}+\mathrm{RT} \ln \mathrm{c}
$$

After determining the relationship between the chemical potential and the concentration we return to the minimization of the free energy. The change in free energy of the whole system is given by the sum of the changes in free energy of the two phases. Phase one is designated by the single prime and 
phase two by the double prime notation and, in the last step, the conservation of particles $d n^{\prime}=-d n^{\prime \prime}$ is used:

$$
d G=d G^{\prime}+d G^{\prime \prime}=\mu^{\prime} d n^{\prime}+\mu^{\prime \prime} d n^{\prime \prime}=\left(\mu^{\prime}-\mu^{\prime \prime}\right) d n^{\prime}
$$

The equilibrium condition, $\mathrm{dG}=0$, yields

$$
\frac{\left(\mu_{0}^{\prime}-\mu_{0}^{\prime \prime}\right)}{R T}=\ln c^{\prime \prime}-\ln c^{\prime}=\ln \frac{c^{\prime \prime}}{c^{\prime}}
$$

The molar partition coefficient $P_{c}$ is therefore related to a characteristic change of free energy, $\Delta G_{0}$, the so called free energy of transfer

$$
\mathrm{P}_{\mathrm{c}}=\frac{\mathrm{c}^{\prime \prime}}{\mathrm{c}^{\prime}}=\mathrm{e}^{\left(\mu_{0}^{\prime}-\mu_{0}^{\prime \prime}\right) / \mathrm{RT}}=\mathrm{e}^{-\Delta \mathrm{G}_{0} / \mathrm{RT}}
$$

Equation 46 describes the connection between the partition coefficient $P_{c}$, an experimentally measurable quantity, and the free energy of transfer - the energy needed to transfer one mole of a compound from one phase to the other phase. For an ideal partitioning system the characteristic free energy of transfer can therefore be determined experimentally. Since the partition coefficients are commonly given as decadic logarithms, we obtain

$$
\Delta G_{0}=-R T \cdot \ln P_{c}=-R T(\ln 10)^{-1} \log P_{c}
$$


Partition coefficients are sometimes defined as a ratio of molar fractions $\left(\mathrm{P}_{\mathrm{x}}\right)$ instead of a ratio of concentrations $\left(P_{c}\right)$. These two partition coefficients are related by ${ }^{8}$

$$
P_{x}=\frac{x^{\prime \prime}}{x^{\prime}}=\frac{c^{\prime \prime} \cdot V_{\text {molar }}^{\prime \prime}}{c^{\prime} \cdot V_{\text {molar }}^{\prime}}=P_{c} \cdot \frac{V_{\text {molar }}^{\prime \prime}}{V_{\text {molar }}^{\prime}}
$$

where $\mathrm{x}^{\prime}$ and $\mathrm{x}$ " are the molar fractions of the partitioning compound and $\mathrm{V}^{\prime}$ molar and V" molar the molar volumes of the media.

Generalization to a system with several solutes results in independent partition coefficients as long as the compounds do not interact with each other. This independence of partition coefficients has been used when measuring octanol-water partition coefficients of several chlorophenols simultaneously (Schellenberg et al., 1984).

\subsubsection{The Octanol-Water System}

\section{a) Description of the Bulk Phases}

When performing the experiments, octanol and water phases are no longer pure. Water was found to be highly soluble in octanol, whereas octanol has a small solubility in water. The equilibrium concentrations of water in octanol reported in literature are 1.7 $\mathrm{M}$ according to Miller et al. (1985) and 2.3 $\mathrm{M}$ according to

\footnotetext{
${ }^{8}$ We used the approximation $x=\frac{n_{A}}{n_{A}+n_{\text {medium }}} \approx \frac{n_{A}}{n_{\text {medium }}}$ for diluted solutions, where $\mathrm{n}_{\mathrm{A}}$ is the number
} of moles of the partitioning compound and $n_{\text {medium }}$ the number of moles of the solvent. 
Leo et al. (1971 In contrast, octanol solubility in water is low, about $4.5 \mathrm{mM}$ (Leo et al., 1971).

The effect of octanol solubility in water can be neglected with regard to volume changes ${ }^{9}$. But the small volume of octanol in the aqueous phase may still alter the aqueous solubility of 2,4,6-TrCP. The solubilities of DDT and hexachlorobenzene, two hydrophobic compounds, were, for example, enhanced by 160 and $80 \%$, respectively to pure water (Chiou et al., 1982). On the other hand, no significant variation in solubility as a result of dissolved octanol in water was found for several other hydrophobic compounds (Miller et al., 1985). The effect of water solubility in octanol cannot be neglected, a $1.7 \mathrm{M}$ concentration corresponds to a change in molar volume ${ }^{10}$ from pure octanol $=$ $157 \mathrm{ml} /$ mole to $127 \mathrm{ml} /$ mole for saturated octanol, or a mole ratio of water to octanol of $1 / 3.5$. The volume changes due to equilibration of water and octanol phases cannot be neglected, though it was found that the saturation of water in the octanol phase gives no significant differences in solubility between octanol saturated water and pure water for several hydrophobic compounds (Miller et al, 1985). The degree of saturation has also been seen to depend on the ionic strength of a solution and, by the use of buffers, on the $\mathrm{pH}$. This variance in saturation occurs only for differences in salt which are quite high. By choosing the right experimental set-up, they can normally be neglected (Westall et al, 1990).

The effect of saturated phases on the thermodynamics in our experiments is considered to be constant due to our experimental set-up. We treat the

\footnotetext{
${ }^{9} 4.5 \mathrm{mM}$ octanol concentration in water corresponds to $0.7 \mathrm{ml}$ of octanol in 11 of octanol saturated water.

${ }^{10}$ The calculation of molar volume for the mixed state was done by assuming no volume change on mixing.
} 
saturated octanol and saturated water phases as phases of constant composition which are not affected by the partitioning.

\section{b) Distribution of Ionized and Un-ionized Species Between the Two Phases}

The derivation of the molar partition coefficient $\mathrm{P}_{c}$, Eq. 46, was done under the assumption that only one species or non-interacting species partition between the two phases. The existence of both ionized and un-ionized, neutral species, of 2,4,6-TrCP in the octanol-water system causes a complication. The goal of our experiment was to determine the partitioning of 2,4,6-TrCP as a function of $\mathrm{pH}$ and the individual partition coefficients for ionized $\left(\mathrm{A}^{-}\right)$and neutral species (HA). If we assume that ionized and un-ionized species are only coupled by the

$\mathrm{pH}$ and behave apart of that as independent solutes, the ideal partitioning can be applied to both of them.

The individual partition coefficients are not directly measurable but can be related to a measurable quantity, the total distribution coefficient of 2,4,6TrCP between octanol and water. The total distribution coefficient, D, is given by

$$
\mathrm{D}=\frac{[\mathrm{HA}]_{\mathrm{oc}}+\left[\mathrm{A}^{-}\right]_{\mathrm{oc}}}{[\mathrm{HA}]_{\mathrm{aq}}+\left[\mathrm{A}^{-}\right]_{\mathrm{aq}}}
$$

where $[\mathrm{HA}]_{\mathrm{oc}}$ and $\left[\mathrm{A}^{-}\right]_{\mathrm{oc}}$ are the concentrations of neutral and ionized 2,4,6$\mathrm{TrCP}$ in octanol, and $[\mathrm{HA}]_{\mathrm{aq}}$ and $[\mathrm{A}]_{\mathrm{aq}}$ are those in water. The individual partition coefficients are defined according to 


$$
\begin{gathered}
g_{H A}=\frac{[H A]_{o c}}{[H A]_{a q}} \\
g_{A}=\frac{\left[A^{-}\right]_{o c}}{\left[A^{-}\right]_{a q}}
\end{gathered}
$$

where $\mathrm{g}_{\mathrm{HA}}$ is the partition coefficient of the neutral species and $\mathrm{g}_{\mathrm{A}}$ the partition coefficient of the ionized species. $\left[\mathrm{A}^{-}\right]_{\mathrm{aq}}$ and $[\mathrm{HA}]_{\mathrm{aq}}$ are related to each other through the definition of $\mathrm{pKa}$ and $\mathrm{pH}$. The $\mathrm{pKa}$ in water is defined as ${ }^{11}$

$$
p K a=-\log \frac{\left[\mathrm{H}_{3} \mathrm{O}^{+}\right]\left[\mathrm{A}^{-}\right]_{a q}}{[H A]_{a q}}
$$

which yields together with $\mathrm{pH}=-\log \left[\mathrm{H}_{3} \mathrm{O}^{+}\right]$

$$
\left[A^{-}\right]_{a q}=[H A]_{a q} 10^{p H-p K a}
$$

Substituting this equation and the definitions of $\mathrm{g}_{\mathrm{A}}$ and $\mathrm{g}_{\mathrm{HA}}$ into Eq. 49 results in

$$
\mathrm{D}=\frac{\mathrm{g}_{\mathrm{HA}}+\mathrm{g}_{\mathrm{A}} \cdot 10^{\mathrm{pH}-\mathrm{pKa}}}{1+10^{\mathrm{pH}-\mathrm{pKa}}}
$$

This equation relates the measurable quantities $\mathrm{D}, \mathrm{pH}$ and $\mathrm{pKa}$ to the unknown $\mathrm{g}_{\mathrm{A}}$ and $\mathrm{g}_{\mathrm{HA}}$. The individual partition coefficients $\mathrm{g}_{\mathrm{A}}$ and $\mathrm{g}_{\mathrm{HA}}$ can be obtained

\footnotetext{
${ }^{11}$ Activity coefficients are set equal to unity.
} 
from the fit of Eq. 54 to the measured $\mathrm{pH}$ dependence of the total distribution coefficient $\mathrm{D}$. For $\mathrm{pH} \ll \mathrm{pKa}$ the distribution coefficient $\mathrm{D}$ converges to $\mathrm{g}_{\mathrm{HA}}$ and to $\mathrm{g}_{\mathrm{A}}$ for $\mathrm{pH} \gg \mathrm{pKa}$. Furthermore, measurements of the total distribution coefficient $\mathrm{D}$ at the limiting $\mathrm{pH}$ values offer, an independent way to determine $\mathrm{g}_{\mathrm{A}}$ and $\mathrm{g}_{\mathrm{HA}}$, in addition to nonlinear fit, Eq. 54.

\subsubsection{The Water-Membrane System}

The partitioning between water and membrane is often described with a linear partition coefficient, $\beta_{X}$, which relates the surface density of the compound $X$ in the membrane, $(\mathrm{X})_{\mathrm{m}}$, to its bulk aqueous concentration, $[\mathrm{X}]_{\mathrm{aq}}$ :

$$
\beta_{X}=(X)_{m} /[X]_{a q}
$$

This linear partition coefficient is a useful tool to describe adsorption measurements to membranes. ${ }^{12}$ To compare the water-membrane system with the octanol-water system a bulk partition coefficient for the membrane-water system is needed.

A bulk partition coefficient, $\gamma_{X}$ for the membrane-water system can be defined as

$$
\gamma_{X}=\left\langle[X]_{m}\right\rangle /[X]_{a q},
$$

\footnotetext{
${ }^{12}$ Adsorption of ionized chlorophenols was determined from changes of electrophoretic mobility of phophatidylcholine liposomes (Blochel, 1992)
} 
where $\left\langle[X]_{m}\right\rangle$ is the average membrane concentration of $X$

$$
\left\langle[X]_{m}\right\rangle=\frac{\int_{0}^{t}[X(z)]_{m} \cdot d z}{t},
$$

where $[\mathrm{X}(\mathrm{z})]_{\mathrm{m}}$ denotes the local concentration, inside the membrane, at a distance $\mathrm{z}$ from the surface and $\mathrm{t}$ denotes the membrane thickness.

Using the symmetry of a bilayer and the definition of the linear partition coefficient, the bulk partition coefficient can also be expressed as

$$
\gamma_{X}=2 \beta_{X} / t
$$

The bulk partition coefficient $\gamma_{X}$ is related to the free energy of transfer of the species X. Therefore, $\log \left(\gamma_{\mathrm{X}}\right)$ is proportional to the free energy of transfer from the bulk aqueous phase into the membrane. 


\subsection{Spectrophotometric Measurements}

Molecules which can be excited to higher energy levels with visible or ultraviolet light can be detected by means of ultraviolet/visible (UV/VIS) spectrophotometry. Chlorophenols, as other conjugated organic compounds, absorb electomagnetic energy on their transition to higher energy levels. The quantum of electromagnetic energy is:

$$
\mathrm{E}=\mathrm{h} v=\frac{\mathrm{hc}}{\lambda}
$$

On return to the ground state, the quantized radiant energy needed for the excitation of the molecules is set free and transformed into other energy forms, mostly thermal energy. Because of this transformation the different energy states of molecules can be observed by means of a spectrophotometer.

Transitions between different energy levels in the range of ultraviolet and visible light correspond basically to changes in the electronic energy though the total energy of a molecule depends on the sum of electronic, vibrational and rotational energies. The magnitude of vibrational and even rotational energies is much smaller than the electronic energy and causes only a splitting in the electronic energy levels, as shown in Figure 13, for a diatomic molecule. This splitting cannot be observed by conventional UV/VIS spectrophotometry because the resolution of spectrophotometers is limited. Instead of discrete absorption lines one observes absorption bands. The characteristic electron transition energies and their transition probabilities result in a characteristic spectrum which can be used for qualitative and quantitative analysis. 


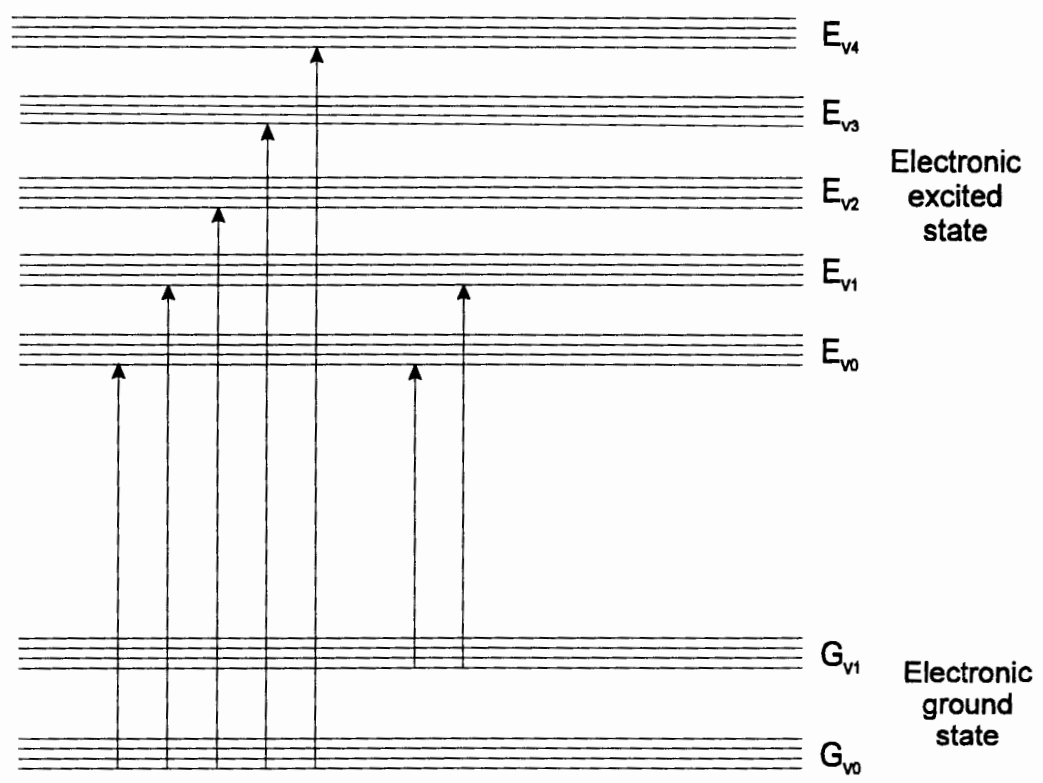

Figure 13. Energy level diagram of a diatomic molecule. The electronic ground state is labeled with $G_{v i}$, the electronic excited state with $E_{v i}$, where $i=$ $0,1,2,3$ and 4 . The vibrational states designated vi, split into different levels due to the rotational energies as indicated by the individual lines within each vibrational band (Silverstein, 1991).

\subsubsection{Lambert-Beer's-Law}

In our experiments we used quantitative analysis of spectra to determine the concentration of solutes in octanol and water phases. The concentration of a solute can be obtained from Lambert-Beer's law,

$$
I(x)=I(0) e^{-A}
$$


where $I(0)$ is the intensity of the radiant energy striking the sample, $I(x)$ the intensity of the radiation emerging from the sample after passing through a path length $x$ and $\mathrm{A}$ is the absorbance. The absorbance depends, in turn, on the extinction coefficient of the solute, $\varepsilon$, its concentration, $c$, and the path length, $\mathrm{x}$.

$$
A=\varepsilon c x,
$$

The probability of the interaction between the radiant energy and the molecules increases with increasing concentration and causes an increase in the light absorption. The extinction coefficient is a characteristic constant of the solute-solvent system. Lambert-Beer's law is applicable to relatively transparent solvents and for solute concentrations at which solute-solute interactions are negligible. The absorbance of the whole sample can be expressed as

$$
\begin{gathered}
A=A_{\text {solv }}+A_{\text {solute }} \\
A_{\text {solv }}=\varepsilon_{\text {solv }} c_{\text {solv }} x \text { and } A_{\text {solute }}=\varepsilon_{\text {solute }} c_{\text {solute }} x
\end{gathered}
$$

The total absorbance is the sum of solvent and solute absorbances. Absolute absorbance of the solute, $A_{\text {solute, }}$ can be obtained by substracting the absorbance of the pure solvent, $A_{\text {solv }}$, from the total absorbance. 


\subsubsection{Solvent Effects}

The solvent, does in fact, have a great influence on the UV/VIS spectrum of the solute due to solvent-solute interactions. The acidity of the solvent determines the degree of dissociation of ionizable solutes, such as chlorophenols, and changes, in this way, their electronic configuration. This is reflected by changes of the UV/VIS absorption spectra.

Another type of solvent effect is related to the polartiy of the solvent. Shifts in absorption spectra are observed as a function of the polarity of the solvent. An increase in polarity of the solvent will cause a bathochromic shift (shift to longer wavelengths) for $\pi \rightarrow \pi^{*}$ absorption bands, and a hypsochromic shift (shift to shorter wavelengths) for $n \rightarrow \pi^{*}$ absorption bands.

Furthermore, formation of micelles in the solvent gives rise to scattering effects which also alter the spectrum of the solute measured by a spectrophotometer. The intensity of scattering depends on the size of the micelles. The scattering is prominent at wavelengths of the same order as the size of the micelles and the intensity of light scattering increases with decreasing wavelength. 


\section{$5 \quad$ Materials and Methods}

\subsection{Chemicals}

2,4,6-Trichlorophenol (98\%) and 1-octanol (99+\%), HPLC grade, were obtained from Aldrich Chemical Company (Milwaukee, WIS). Boric acid and potassium phosphate dibasic were bought from Mallinckrodt (St. Louis, MO), potassium chloride from EM Science (Cherry Hill, NJ) and potassium citrate from MCB Manufacturing Chemists (Cincinnati, Ohio). They were all used without further purification. The aqueous solutions were prepared with deionized water.

\subsection{Instruments and their Specifications}

The weighing of chemicals for preparation of all stock solutions was done using a balance from Mettler Instrument, Type H16 (Hightstown, NJ). The estimated accuracy of this model is $\pm 0.1 \mathrm{mg}$.

All $\mathrm{pH}$ measurements were done using a micro computer based $\mathrm{pH}$ meter, model 6072, from Jenco Electronics (Taipei, Taiwan). This $\mathrm{pH}$ meter has a $\pm 0.01 \mathrm{pH}$ accuracy.

The spectrophotometer used for all concentration measurements was Model DU-7HS from Beckman Instruments (Irvine, CA). The following specifications of this spectrophotometer are important for the determination of 
the accuracy of our measurements. The specifications are as follows: $\pm 0.5 \mathrm{~nm}$ wavelength accuracy, $\pm 0.5 \%$ photometric accuracy, baseline drift $<0.003$ $\mathrm{Abs} / \mathrm{hr}$ and $\pm 0.001 \mathrm{Abs}$ baseline flatness.

Throughout the study we used well matched pairs ${ }^{13}$ of quartz cuvettes as sample cells. For the buffer phase we used $1 \mathrm{~mm}^{14}$ and $50 \mathrm{~mm}$ cuvettes from Spectrocell Corporation (Oreland, PA) and $10 \mathrm{~mm}$ cuvettes from Pyrocell Manufacturing Co., Inc (Westwood, NJ). Spectra in the octanol phase were obtained with $10 \mathrm{~mm}$ cuvettes from Spectrocell Corporation (Oreland, PA).

The analysis of all data and the graphics were done with Axum V.4 for Windows.

\subsection{Preparation of Octanol and Aqueous Phases}

The aqueous phase, also called buffer phase, consists of a phosphoric acid/potassium citrate/boric acid $(2 \mathrm{mM} / 2 \mathrm{mM} / 0.5 \mathrm{mM})$ buffer with a salt concentration of $0.03 \mathrm{M} \mathrm{KCl}$. The solution was prepared from a 100 times concentrated buffer solution and a $2 \mathrm{M} \mathrm{KCl}$ stock solution. The addition of $\mathrm{KCl}$ was needed to achieve the same experimental conditions as in the membrane adsorption measurements (Blochel, 1992) and to obtain, for all experiments, the same potassium concentration ${ }^{15}$.

\footnotetext{
${ }^{13}$ Well matched pairs means in this context that the absorbance difference of such a pair should be less than 0.005 .

${ }^{14}$ The path lengths of the $1 \mathrm{~mm}$ cuvettes were actually determined to be $\mathrm{L}=1.12+0.01 \mathrm{~mm}$ and $\mathrm{L}=$ $1.11+0.02 \mathrm{~mm}$.

${ }^{15}$ The partitioning of the ionized species of 2,4,6-Trichlorophenol is strongly dependent on the $\mathrm{K}^{+}$concentration in the aqueous phase (Westall, 1985).
} 
Octanol and buffer phases were preequilibrated before all experiments. This was necessary since octanol and water are partially miscible as described in 4.1.2 a. The miscibility affects the volume and the spectroscopic properties of the octanol and buffer phases. These two phases were mutually equilibriated before all experiments and an octanol/water ratio of 1:5 was used. Octanol and buffer phases were mixed for one hour with a Wrist-Action shaker model BB from Burell Corp. (Pittsburgh, PA) in $250 \mathrm{ml}$ polypropylene centrifuge bottles, and afterwards centrifuged at $1500 \mathrm{~g}$ with a IEC DPR-600 centrifuge.

The separation of the two phases was performed by removing the octanol-phase from the top of the bottles with a syringe. The buffer phase was sucked out with a $100 \mathrm{ml}$ pipette. Both the octanol and the buffer phases were collected from each batch ${ }^{16}$ and measured spectrophotometrically using the corresponding unsaturated phases as background. Spectrophotometric measurements were necessary to examine the effect of phase saturation and to varify that the method of equilibration was reproducible.

It was found that the buffer phase is only slightly affected by saturation with octanol, see Figure 14. The difference in absorbance between the saturated and the unsaturated phases was always smaller than 0.01 . The octanol phase, see Figure 15, always shows two peaks, one at about $225 \mathrm{~nm}$ with absorbance varying between 0.1 and 0.6 and the other at about $276 \mathrm{~nm}$ with absorbance varying between 0.04 and 0.09 . The reason for these peaks is unknown, the absorbance at these wavelength occurs probably as a result of the formation of water micelles in the octanol phase. The variation of the absorbance between samples can be explained as a function of the variation in the concentration of water micelles in octanol from batch to batch.

\footnotetext{
${ }^{16}$ Each batch consists of about 4-6 centrifuge bottles.
} 


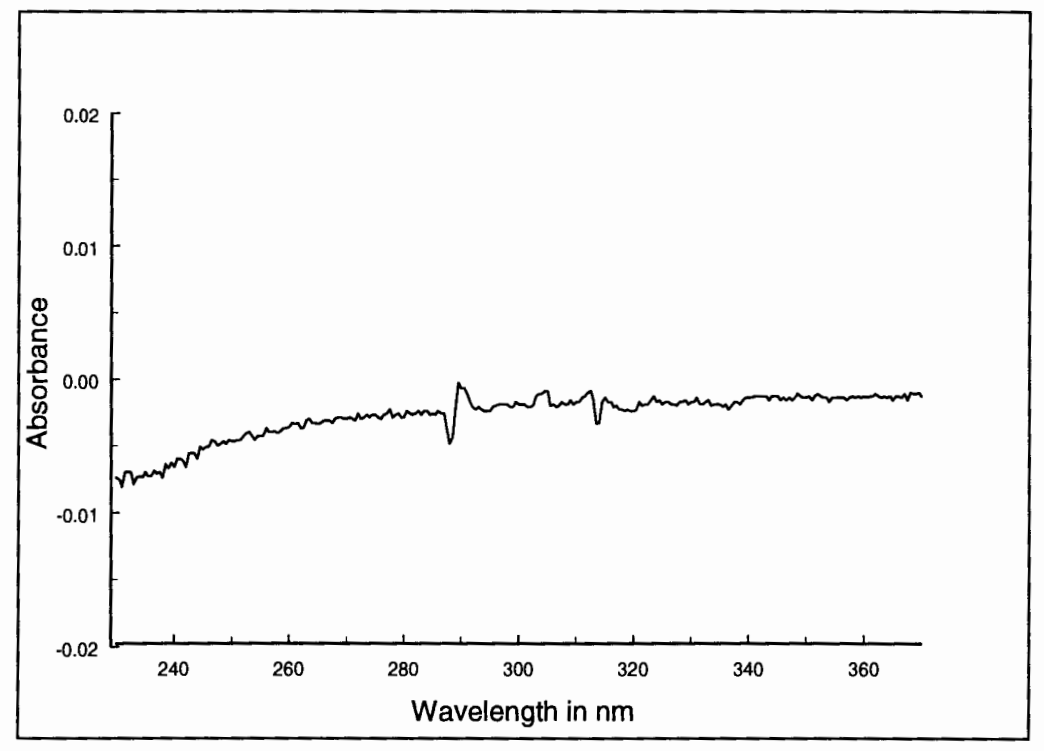

Figure 14. Typical spectrum of the saturated buffer phase, measured against pure buffer.

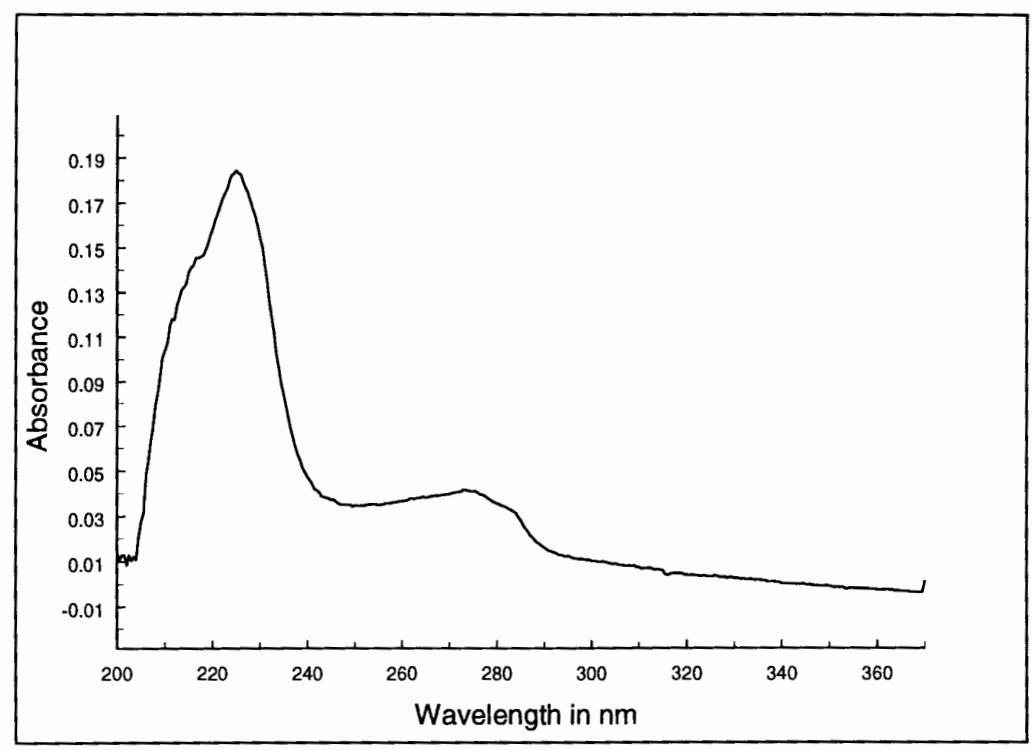

Figure 15. Typical spectrum of the saturated octanol phase, measured against pure octanol. 


\subsection{Extinction Coefficients}

The distribution of 2,4,6-TrCP between the octanol phase and the buffer phase was determined by spectroscopic determination of the concentration of $2,4,6$ $\mathrm{TrCP}$ in the octanol and the buffer phases. Therefore, as a first step, the extinction coefficients of 2,4,6-TrCP in octanol-saturated buffer and watersaturated octanol were determined.

The absorbance of $2,4,6-\operatorname{TrCP}$ is $\mathrm{pH}$ dependent due to its ionization. The neutral form has an absorbance peak at $293 \mathrm{~nm}$ and in its ionized form at 311.5 $\mathrm{nm}$. For all concentration determinations in the aqueous phase, 2,4,6-TrCP was converted into its ionized form by adjusting the $\mathrm{pH}$ to about $10-11 .{ }^{17}$ Due to this simplification of the experimental protocol it was necessary to determine the extinction coefficients only for the ionized species.

The applicability of Lamberts-Beer's law was tested for sample concentrations between 40 and $200 \mu \mathrm{M}$. Those were prepared from four different stock solutions (1-3 mM). The stock solutions were prepared with unsaturated buffer because at such high 2,4,6-TrCP concentrations 2,4,6-TrCP tends to aggregate with octanol present in the aqueous phase. Saturated buffer was then used for the dilution of the stock solutions to the different sample concentrations. The sample solutions were titrated with $\mathrm{KOH}$ to $\mathrm{pH} \sim 10-11$ and the absorbance determined in $1 \mathrm{~cm}$ cuvettes.

The extinction coefficient in saturated octanol was measured for sample concentrations between 0.1 and $0.6 \mathrm{mM}$, prepared from stock solutions 5-6 $\mathrm{mM}$.

\footnotetext{
${ }^{17}$ The $\mathrm{pKa}$ of 2,4,6- $\mathrm{TrCP}$ is 6.1 (Schellenberg, 1984), a titration to $\mathrm{pH} \sim 10-11$ corresponds therefore to more than $99.9 \%$ ionization of $2,4,6-\mathrm{TrCP}$.
} 


\subsection{Partitioning}

The partitioning experiment was performed with two different sets of preequilibrated phases. The first set consisted of three different batches. Saturated octanol and aqueous phases from these three batches were respectively collected and used for as follows:

First, $100 \mathrm{ml}$ of a $20 \mathrm{mM} \mathrm{2,4,6-TrCP}$ of water-saturated octanol solution was prepared. Then for each partitioning experiment we used $5 \mathrm{ml}$ of this $2,4,6-$ TrCP saturated octanol stock solution to which we added $25 \mathrm{ml}$ of $\mathrm{pH}$ adjusted saturated buffer. The $\mathrm{pH}$ of the saturated buffer phase was set to values between 4.91 and 12.77 by the addition of $\mathrm{HCl}$ or $\mathrm{KOH}$. The sample containing $25 \mathrm{ml}$ of the $\mathrm{pH}$ adjusted buffer and the $5 \mathrm{ml}$ of the $20 \mathrm{mM} 2,4,6-\operatorname{TrCP}$ saturated octanol were mixed in $50 \mathrm{ml}$ glass tubes, shaked for one hour (with the same shaker used for the phase saturation) and were afterwards centrifuged at $1500 \mathrm{~g}$ for one hour in a Safeguard centrifuge from Clay-Adams Inc. (New York, NY). Since the samples warm up during the centrifugation step it was necessary to postpone the analysis an hour after centrifugation that the samples are reequilibrated at the test temperature.

The sample analysis protocol included $\mathrm{pH}$ and 2,4,6- $\mathrm{TrCP}$ concentration measurements of the buffer phase. The $\mathrm{pH}$ of the buffer phase had to be measured after the partitioning since 2,4,6-TrCP is a weak acid and changes the $\mathrm{pH}$. The final $\mathrm{pH}$ ranged from 4.98 to 12.72 . The concentration of $2,4,6-\operatorname{TrCP}$

in the buffer was determined after the $\mathrm{pH}$-measurement and after titrating the sample to $\mathrm{pH} \sim 10-11$. The three different cuvette pairs $(1,10,50 \mathrm{~mm})$ were used depending on the concentration of 2,4,6-TrCP. 
A second set of partitioning experiments were performed with only one

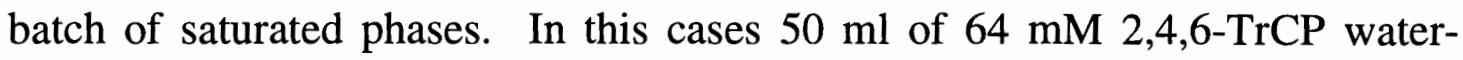
saturated octanol solution was used and the final $\mathrm{pH}$ varied between 3.49 and 7.72. Apart from that the preparation and measurement methods were the same as in the first run.

For both runs the experimental protocol was designed to minimize the errors involved in all measurements: (a) The number of sets of preequilibrated phases used to perform the experiment was reduced to two, (b) the ratio of octanol to buffer phase was the same, $5: 1$, for both the partitioning experiment as well as the phase presaturation. ${ }^{18}$ (c) $2,4,6-\operatorname{TrCP}$ stock solutions in octanol, rather than in buffer, were used because of the higher solubility of 2,4,6-TrCP in octanol which made it possible to weigh manageable amounts of $2,4,6-\operatorname{TrCP}$ (about 390 and $630 \mathrm{mg}$ ) and thus to obtain well defined stock solution. The use of stock solutions has the additional advantage of maintaining the total concentration of $2,4,6-\operatorname{TrCP}$ constant which makes it then possible to obtain a meaningful plot of 2,4,6-TrCP concentrations in the two phases versus $\mathrm{pH}$, in addition to the $\mathrm{pH}$ dependence of the total distribution coefficient.

\footnotetext{
${ }^{18}$ A possible change in the degree of saturation due to different saturation methods is therefore nearly excluded.
} 


\section{Results}

\subsection{Extinction Coefficients}

\subsubsection{Results}

The extinction coefficients of 2,4,6-Trichlorophenol in buffer $\left(\varepsilon_{\text {buf }}\right)$ and octanol $\left(\varepsilon_{o c}\right)$ have been measured for several concentrations using $1 \mathrm{~cm}$ cuvettes. Characteristic absorbance spectra for 2,4,6-TrCP are shown in Figure 16 for octanol and in Figure 17 for buffer. Table 1 lists the values for the extinction coefficient obtained for the buffer and Table 2 for the octanol. The average extinction coefficient in the buffer phase was calculated, including the measured values in pure buffer. No measurable differences between $\varepsilon_{\text {buf }}$ in the pure and the saturated buffer could be detected. The average peak extinction coefficients are:

$4800 \pm 1501 / \mathrm{Mcm}$ (at $\lambda=311.5 \mathrm{~nm}$ ) for ionized $2,4,6-\operatorname{TrCP}$ in the octanol saturated buffer, and

$2900 \pm 701 / \mathrm{Mcm}$ (at $\lambda=297.5 \mathrm{~nm}$ ) for un-ionized $2,4,6-\operatorname{TrCP}$ in the water saturated octanol. 


\subsubsection{Error Calculations}

The standard deviation or the statistical error of both extinction coefficients is about 3\% and can be explained by the errors involved in both the sample preparation and in the measurements of the absorbance. Error calculation was done to gain insight into the different origins of errors. The maximum error of the extinction coefficients was calculated using the following general relation for the absolute maximum errror:

$$
\Delta F= \pm\left(\left|\frac{\partial F}{\partial x} \Delta x\right|+\left|\frac{\partial F}{\partial y} \Delta y\right|+\ldots\right)
$$

$\Delta \mathrm{F}$ stands for the maximum absolute error of a measured quantity $\mathrm{F} ; \Delta \mathrm{x}, \Delta \mathrm{y}, \ldots$ are the estimated errors of the measured values.

The error of weighing was estimated to be $\pm 0.1 \mathrm{mg}$ and the error of all volumetric measurements to be 1\%. Applying Eq. 63, the maximum error resulting from weighing and the different volumetric measurements varies between $3.4 \%$ and $4.3 \%$. The maximum error resulting from absorbance measurements (estimated error \pm 0.005 ) varies beteween $0.4 \%$ and $2.4 \%$. Therefore, the total errors range from $3.7 \%$ to $6.3 \%$ and are consistent with the the magnitude of the standard deviations of extinction coefficients. 


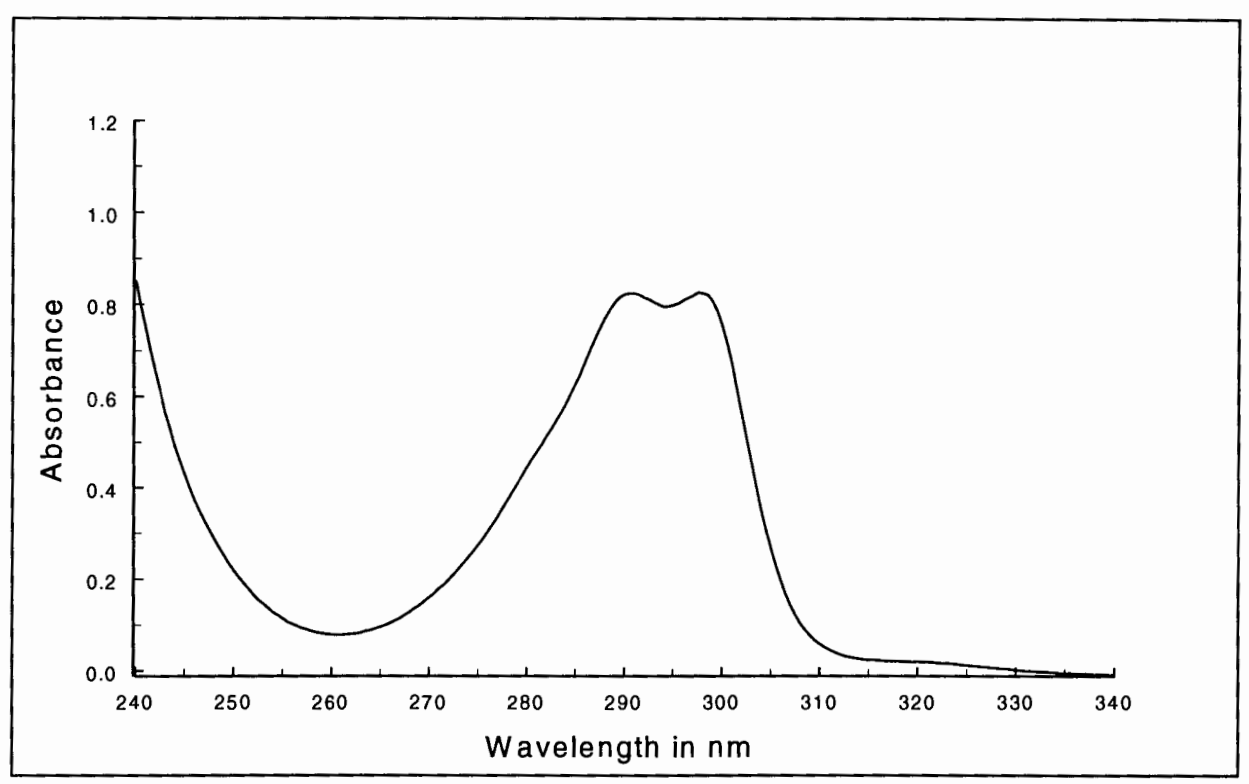

Figure 16. Spectrum of un-ionized $2,4,6-\operatorname{TrCP}(285 \mu \mathrm{M})$ in water-saturated octanol.

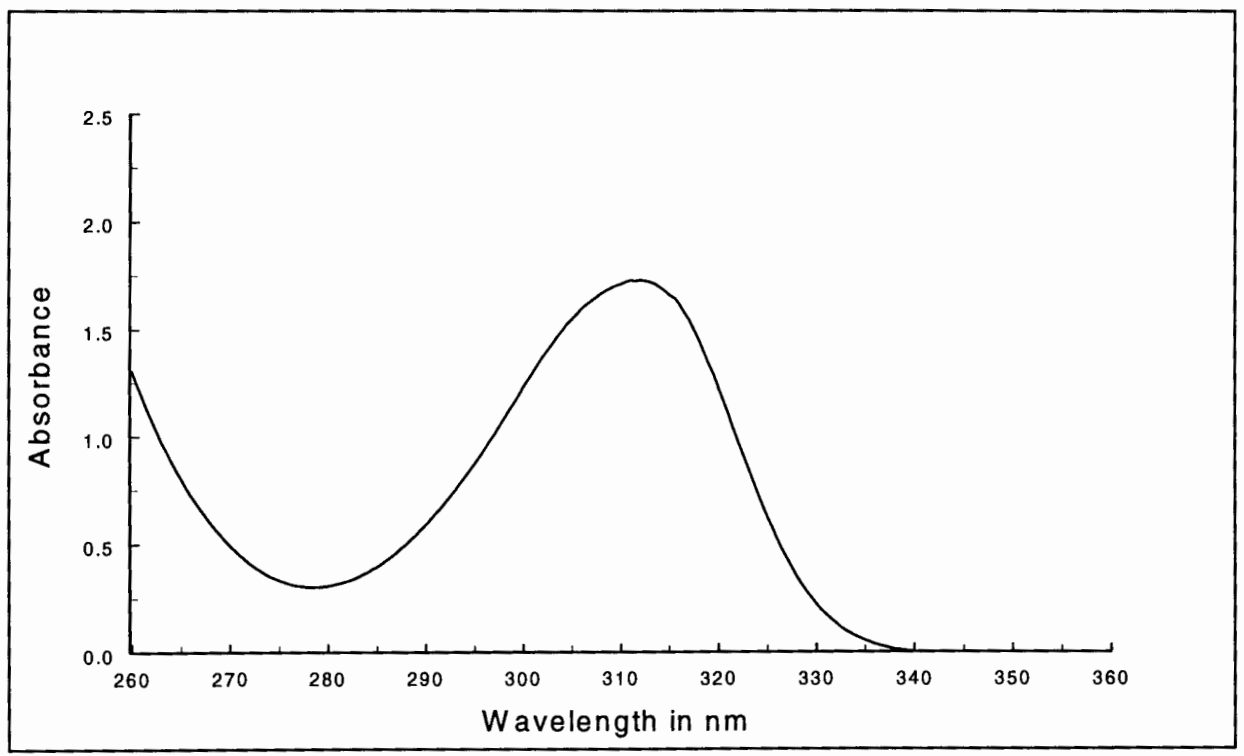

Figure 17. Spectrum of ionized $2,4,6,-\operatorname{TrCP}(36 \mu \mathrm{M})$ in octanol-saturated buffer. 


\begin{tabular}{|l|l|l|l|l|l|}
\hline solvent & $\begin{array}{l}\text { stock } \\
\text { concentration } \\
{[\mathrm{mM}]}\end{array}$ & $\begin{array}{l}\text { sample } \\
\text { concentration } \\
{[\mu \mathrm{M}]}\end{array}$ & $\mathrm{pH}$ & $\begin{array}{l}\text { absorbance } \\
\text { at } \lambda=311.5 \\
\mathrm{~nm}\end{array}$ & $\begin{array}{l}\text { extinction } \\
\text { coefficient } \\
{[1 / \mathrm{M} \cdot \mathrm{cm}]}\end{array}$ \\
\hline $\mathrm{B}-3$ & 2.73 & 109 & $\sim 10$ & 0.524 & 4800 \\
\hline $\mathrm{B}-3$ & 2.73 & 109 & $\sim 10$ & 0.534 & 4900 \\
\hline $\mathrm{B}-3^{*}$ & 1.08 & 43 & 10.44 & 0.213 & 5000 \\
\hline $\mathrm{B}-3^{*}$ & 1.08 & 86 & 10.14 & 0.418 & 4900 \\
\hline $\mathrm{B}-3^{*}$ & 1.22 & 120 & 10.28 & 0.599 & 5000 \\
\hline $\mathrm{B}-3^{*}$ & 1.22 & 168 & 10.42 & 0.842 & 5000 \\
\hline $\mathrm{B}-3^{*}$ & 1.22 & 72 & 10.44 & 0.356 & 4900 \\
\hline $\mathrm{B}-3^{*}$ & 1.09 & 54 & 10.30 & 0.250 & 4700 \\
\hline $\mathrm{B}-3^{*}$ & 1.09 & 107 & 10.20 & 0.494 & 4600 \\
\hline B-3* & 1.09 & 213 & 10.72 & 0.989 & 4600 \\
\hline \multicolumn{2}{|l|}{ average extinction coefficient $=4800 \pm 150(\lambda=311.5 \mathrm{~nm})$} \\
\hline
\end{tabular}

Table 1. Extinction coefficients of 2,4,6-Trichlorophenol in buffer; B-3 designates unsaturated aqueous phase, B-3* the octanol-saturated aqueous phase.

\begin{tabular}{|l|l|l|l|l|}
\hline solvent & $\begin{array}{l}\text { stock } \\
\text { concentration } \\
{[\mathrm{mM}]}\end{array}$ & $\begin{array}{l}\text { sample } \\
\text { concentration } \\
{[\mu \mathrm{M}]}\end{array}$ & $\begin{array}{l}\text { absorbance } \\
\text { at } \lambda=297.5 \\
\mathrm{~nm}\end{array}$ & $\begin{array}{l}\text { extinction } \\
\text { coefficient } \\
{[1 / \mathrm{M} \cdot \mathrm{cm}]}\end{array}$ \\
\hline Oct* & 5.46 & 328 & .961 & 2930 \\
\hline Oct* & 5.46 & 437 & 1.242 & 2840 \\
\hline Oct* & 5.46 & 546 & 1.572 & 2880 \\
\hline Oct* & 5.46 & 219 & .616 & 2820 \\
\hline Oct* & 5.46 & 328 & .994 & 3030 \\
\hline Oct* & 5.68 & 341 & .997 & 2920 \\
\hline Oct* & 5.68 & 114 & .332 & 2920 \\
\hline \multicolumn{2}{|l|}{ average extinction coefficient $=2900 \pm 70(\lambda=297.5 \mathrm{~nm})$} \\
\hline
\end{tabular}

Table 2. Extinction coefficients of 2,4,6-Trichlorophenol in water-saturated octanol. 


\subsection{Partitioning}

\subsubsection{Results}

The octanol-water partition experiment was performed with two different initial concentrations ${ }^{19}$ of 2,4,6-TrCP in saturated octanol to obtain the $\mathrm{pH}$-dependence of the distribution for the $\mathrm{pH}$ from 3.5 to 12.7. Two initial concentrations of $2,4,6-\operatorname{TrCP}$ in saturated octanol were necesssary in order to obtain data at $\mathrm{pH}<<$ $\mathrm{pKa}$ and $\mathrm{pH} \gg \mathrm{pKa}$.

In most cases the distribution of $2,4,6-\operatorname{TrCP}$ was based on measurements of 2,4,6-TrCP in the buffer phase. In the buffer phase the 2,4,6-TrCP concentration changes, as a function of $\mathrm{pH}$, by several orders of magnitude whereas the concentration in the octanol phase remains about the same. The 2,4,6-TrCP concentrations in the octanol phase were calculated using the mass conservation of $2,4,6-\operatorname{TrCP}$. The mass balance of $2,4,6-\operatorname{TrCP}$ was verified experimentally several times by measuring the concentration of $2,4,6-\operatorname{Tr} \mathrm{CP}$ in the octanol phase. The error in mass balance was random and varied between $1.5 \%$ and $6.9 \%$. Those errors are within the limit of the measurement errors ${ }^{20}$, errors which were about $10 \%$. Therefore, within the limits of the concentration measurement the mass was conserved.

The experimentally measured buffer and octanol concentrations of 2,4,6$\mathrm{TrCP}$ as a function of $\mathrm{pH}$ are shown in Table 4 and Table 5. These data were

\footnotetext{
${ }^{19}$ The initial concentration is the $2,4,6-\mathrm{TrCP}$-concentration in the water-saturated octanol injected into the octanol phase.
}

${ }^{20}$ The measurement errrors were calculated using Eq. 63. 
used to obtain the total distribution coefficient according to Eq. 49. The octanol-water partition coefficients of the ionized, $\mathrm{g}_{\mathrm{A}}$, and the un-ionized species, $\mathrm{g}_{\mathrm{HA}}$, were obtained by fitting the $\mathrm{pH}$ dependence of $\log \mathrm{D}$. A logarithmic fit of $\mathrm{D}$ was used since the relative error, and not the total error, was of physical interest.

It follows from the theory of the ideal partitioning (4.1.1) that the logarithm of partition coefficient is proportional to the free energy of transfer according to Eq. 47. Therefore, the fit of $\log \mathrm{D}$ rather than that of $\mathrm{D}$ itself has greater physical meaning. In the limits of very low $\mathrm{pH}, \mathrm{D} \approx \mathrm{g}_{\mathrm{HA}}$ and in the limits of very high $\mathrm{pH}, \mathrm{D} \approx \mathrm{g}_{\mathrm{A}}$.

The best fit was obtained for the following octanol-water partition coefficients of individual species:

$$
\begin{aligned}
& \mathrm{g}_{\mathrm{A}}=1.42 \pm 0.16 \\
& \mathrm{~g}_{\mathrm{HA}}=4500 \pm 210
\end{aligned}
$$

or expressed in terms of the decadic logarithm:

$$
\begin{aligned}
& \log \left(g_{A}\right)=0.15 \pm 0.05 \\
& \log \left(g_{H A}\right)=3.65 \pm 0.02
\end{aligned}
$$

The $\mathrm{pH}$ dependence of the measured total distribution coefficient $\mathrm{D}$ of $2,4,6$ TrCP is given in Table 3. The experimental results as well as the theoretical predictions of the partition model for the best parameters are shown in Figure 18. Similar data for the concentrations in octanol and buffer phases are 
presented in Table 4 and Table 5 and Figure 19 and 20. The gray lines illustrate the standard deviation of the predicted values.

The plot of the total distribution coefficient for the best parameters was done using Eq. 54. The plot of the concentrations in the octanol and buffer phases was based on the mass balance and the definition of the total distribution coefficient, Eq. 49. The mass balance is given by

$$
\left([\mathrm{HA}]_{\mathrm{aq}}+\left[\mathrm{A}^{-}\right]_{\mathrm{aq}}\right) \cdot \mathrm{vol}_{\mathrm{aq}}+\left([\mathrm{HA}]_{\mathrm{oc}}+\left[\mathrm{A}^{-}\right]_{\mathrm{oc}}\right) \cdot \operatorname{vol}_{\mathrm{oc}}=[\mathrm{HA}]_{\mathrm{input}} \cdot \operatorname{vol}_{\mathrm{oc}}
$$

where $\left([\mathrm{HA}]_{\mathrm{aq}}+\left[\mathrm{A}^{-}\right]_{\mathrm{aq}}\right)$ and $\left([\mathrm{HA}]_{\mathrm{oc}}+\left[\mathrm{A}^{-}\right]_{\mathrm{oc}}\right)$ are the equilibrium concentrations of 2,4,6-TrCP in the buffer and octanol phase, and $[\mathrm{HA}]_{\text {input }}$ the inititial concentration of 2,4,6-TrCP in the octanol phase. The quantities $v^{2} l_{\mathrm{aq}}$ and $\mathrm{vol}_{\mathrm{oc}}$ designate the buffer and octanol volume. The mass balance together with Eq. 49 results in the following expression for the total concentration in the buffer phase

$$
\left([\mathrm{HA}]_{\mathrm{aq}}+\left[\mathrm{A}^{-}\right]_{\mathrm{aq}}\right)=\frac{[\mathrm{HA}]_{\mathrm{input}_{\mathrm{t}}}}{\text { vol }_{\mathrm{aq}} / \operatorname{vol}_{\mathrm{oc}}+\mathrm{D}}
$$

The total concentration in the octanol phase predicted from the partition model

$$
\left([\mathrm{HA}]_{\mathrm{oc}}+\left[\mathrm{A}^{-}\right]_{\mathrm{oc}}\right)=\frac{\mathrm{D} \cdot[\mathrm{HA}]_{\text {input }}}{\operatorname{vol}_{\mathrm{aq}} / \mathrm{vol}_{\mathrm{oc}}+\mathrm{D}}
$$

The value of the partition coefficient of the un-ionized species of 2,4,6- $\operatorname{TrCP}$, obtained in this study, $\log \left(\mathrm{g}_{\mathrm{HA}}\right)=3.65 \pm 0.02$, is in good agreement with 
literature values that range from 3.38 to 3.72 (Schellenberg et al., 1984; Callahan et al., 1979; Xie et al., 1984 and Hansch, 1971). The value of the partition coefficient of the ionized species of 2,4,6-TrCP is several orders smaller compared to that of the un-ionized species. There are no literature values of the octanol-water partition coefficients for the ionized species of 2,4,6-TrCP.

\subsubsection{Error Calculations}

The confidence limits of the partition parameters associated with their standard deviation are shown as grey lines in Figure 18, 19, 20. The maximum experimental errors calculated from Eq. 63 are shown as error bars. The total distribution coefficients are listed in Table 3. The concentration data are summarized in Table 4 and Table 5. The following errors were taken into account in the error calculations:

- standard deviations of the measured extinction coefficients

- errors in the saturated buffer and octanol volumes, these were estimated to be $1 \%$

- error in absorbance measurement, estimated as \pm 0.005 absorbance units

- error in the path length of the cuvettes, estimated as $2 \%$

- errror in weighing (estimated as $\pm 0.1 \mathrm{mg}$ )

In general, the errors of all error sources increase with the decreasing value of $\log \mathrm{D}$. This can be clearly seen in the data in Table 3. The logarithmic fit due to these errors is better at low $\mathrm{pH}$ values rather than at high $\mathrm{pH}$ values. 


\begin{tabular}{|l|l|l|l|l|}
\hline final $\mathrm{pH}$ & $D=\frac{[\mathrm{HA}]_{o c}+\left[\mathrm{A}^{-}\right]_{o c}}{[\mathrm{HA}]_{a q}+\left[\mathrm{A}^{-}\right]_{a q}}$ & $\Delta \mathrm{D}[\%]$ & $\log \mathrm{D}$ & $\Delta \log \mathrm{D}[\%]$ \\
\hline 3.49 & $4.24 \mathrm{e}+3$ & 7.02 & 3.63 & 1.93 \\
\hline 4.74 & $4.43 \mathrm{e}+3$ & 7.05 & 3.65 & 1.93 \\
\hline 4.98 & $3.19 \mathrm{e}+3$ & 9.11 & 3.50 & 2.60 \\
\hline 5.60 & $2.30 \mathrm{e}+3$ & 6.56 & 3.36 & 1.95 \\
\hline 6.10 & $2.92 \mathrm{e}+3$ & 8.81 & 3.47 & 2.54 \\
\hline 6.54 & $1.08 \mathrm{e}+3$ & 6.68 & 3.03 & 2.20 \\
\hline 6.77 & $8.71 \mathrm{e}+2$ & 6.99 & 2.94 & 2.38 \\
\hline 7.13 & $3.74 \mathrm{e}+2$ & 6.02 & 2.57 & 2.34 \\
\hline 7.57 & $1.80 \mathrm{e}+2$ & 8.49 & 2.26 & 3.76 \\
\hline 7.72 & $1.05 \mathrm{e}+2$ & 7.54 & 2.02 & 3.73 \\
\hline 7.76 & $1.06 \mathrm{e}+2$ & 6.72 & 2.03 & 3.31 \\
\hline 7.88 & $7.14 \mathrm{e}+1$ & 6.46 & 1.85 & 3.49 \\
\hline 8.15 & $4.27 \mathrm{e}+1$ & 8.94 & 1.63 & 5.48 \\
\hline 8.66 & $1.68 \mathrm{e}+1$ & 9.05 & 1.23 & 7.83 \\
\hline 9.82 & $2.65 \mathrm{e}+0$ & 20.7 & 0.422 & 48.8 \\
\hline 11.68 & $1.27 \mathrm{e}+0$ & 36.5 & 0.102 & 355 \\
\hline 12.72 & $1.43 \mathrm{e}+0$ & 32.9 & 0.156 & 210 \\
\hline
\end{tabular}

Table 3. Experimental data and calculated error for the $\mathrm{pH}$ dependence of the total distribution coefficient of 2,4,6-Trichlorophenol between octanol and water. 


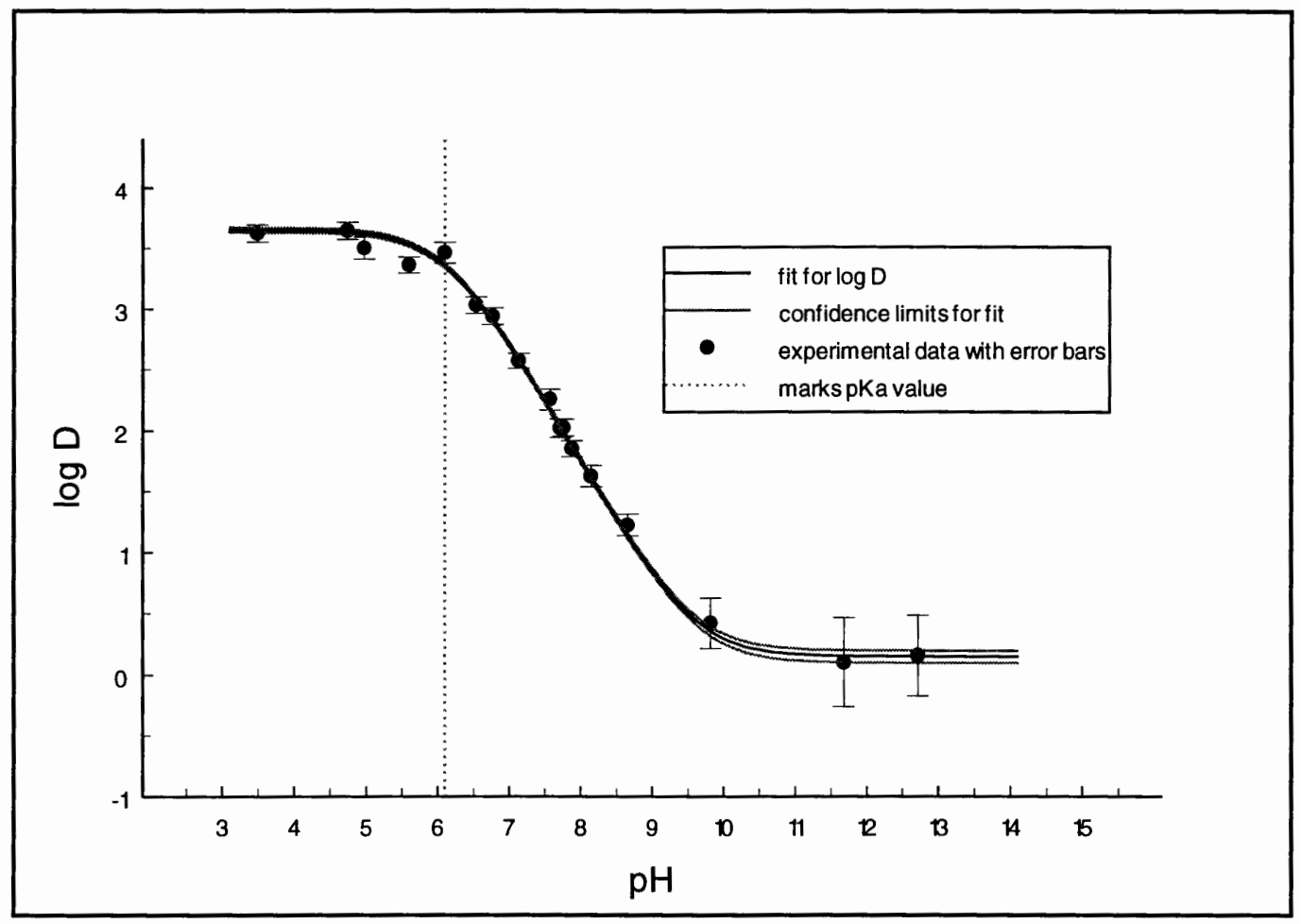

Figure 18. The $\mathrm{pH}$ dependence of the total distribution coefficient $\mathrm{D}$ of 2,4,6-Trichlorophenol between octanol and water. 


\begin{tabular}{|l|l|l|l|}
\hline final $\mathrm{pH}$ & $\begin{array}{l}{[\mathrm{HA}]_{\mathrm{aq}}+[\mathrm{A}-]_{\mathrm{aq}}} \\
{[\mathrm{mM}]}\end{array}$ & $\begin{array}{l}\Delta\left([\mathrm{HA}]_{\mathrm{aq}}+[\mathrm{A}-]_{\mathrm{aq}}\right) \\
{[\%]}\end{array}$ & $\begin{array}{l}{[\mathrm{HA}]_{\mathrm{oc}}+[\mathrm{A}-]_{\mathrm{oc}}[\mathrm{mM}]} \\
\text { calculated with mass balance }\end{array}$ \\
\hline 4.98 & $6.16 \mathrm{e}-3$ & 8.97 & $1.97 \mathrm{e}+1$ \\
\hline 6.10 & $6.74 \mathrm{e}-3$ & 8.66 & $1.97 \mathrm{e}+1$ \\
\hline 6.54 & $1.82 \mathrm{e}-2$ & 6.51 & $1.96 \mathrm{e}+1$ \\
\hline 7.13 & $5.21 \mathrm{e}-2$ & 5.79 & $1.95 \mathrm{e}+1$ \\
\hline 7.76 & $1.77 \mathrm{e}-1$ & 6.20 & $1.88 \mathrm{e}+1$ \\
\hline 7.88 & $2.58 \mathrm{e}-1$ & 5.78 & $1.84 \mathrm{e}+1$ \\
\hline 8.15 & $4.13 \mathrm{e}-1$ & 7.67 & $1.77 \mathrm{e}+1$ \\
\hline 8.66 & $9.02 \mathrm{e}-1$ & 6.40 & $1.52 \mathrm{e}+1$ \\
\hline 9.82 & $2.58 \mathrm{e}-0$ & 5.71 & $6.83 \mathrm{e}+0$ \\
\hline 11.68 & $3.15 \mathrm{e}-0$ & 5.65 & $3.99 \mathrm{e}+0$ \\
\hline 12.72 & $3.02 \mathrm{e}-0$ & 5.65 & $4.40 \mathrm{e}+0$ \\
\hline
\end{tabular}

Table 4. Experimental results. Total aqueous and octanol concentrations of 2,4,6-TrCP obtained for the $20 \mathrm{mM}$ initial concentration of 2,4,6-TrCP in octanol, $\Delta\left([\mathrm{HA}]_{\mathrm{aq}}+[\mathrm{A}-]_{\mathrm{aq}}\right)$ designates the calculated maximum error.

\begin{tabular}{|l|l|l|l|l|}
\hline $\begin{array}{l}\text { final } \\
\mathrm{pH}\end{array}$ & $\begin{array}{l}{[\mathrm{HA}]_{\mathrm{aq}}+[\mathrm{A}-]_{\mathrm{aq}}} \\
\text { in } \mathrm{mM}\end{array}$ & $\begin{array}{l}\Delta\left([\mathrm{HA}]_{\mathrm{aq}}+[\mathrm{A}-]_{\mathrm{aq}}\right. \\
\text { in \% }\end{array}$ & $\begin{array}{l}{[\mathrm{HA}]_{\mathrm{oc}}+[\mathrm{A}-]_{\mathrm{oc}}} \\
\text { in } \mathrm{mM}, \\
\text { calculated } \\
\text { with mass } \\
\text { balance }\end{array}$ & $\begin{array}{l}{[\mathrm{HA}]_{\mathrm{oc}}+[\mathrm{A}-]_{\mathrm{oc}} \text { in }} \\
\mathrm{mM}, \\
\text { experimental } \\
\text { data }\end{array}$ \\
\hline 3.49 & $1.51 \mathrm{e}-2$ & 6.89 & $6.40 \mathrm{e}+1$ & $6.85 \mathrm{e}+1$ \\
\hline 4.74 & $1.44 \mathrm{e}-2$ & 6.92 & $6.40 \mathrm{e}+1$ & $5.87 \mathrm{e}+1$ \\
\hline 5.60 & $2.77 \mathrm{e}-2$ & 6.42 & $6.39 \mathrm{e}+1$ & $6.40 \mathrm{e}+1$ \\
\hline 6.77 & $7.31 \mathrm{e}-2$ & 6.82 & $6.37 \mathrm{e}+1$ & $5.68 \mathrm{e}+1$ \\
\hline 7.57 & $3.46 \mathrm{e}-1$ & 8.09 & $6.23 \mathrm{e}+1$ & $5.74 \mathrm{e}+1$ \\
\hline 7.72 & $5.80 \mathrm{e}-1$ & 6.99 & $6.12 \mathrm{e}+1$ & $5.97 \mathrm{e}+1$ \\
\hline
\end{tabular}

Table 5. Experimental results. Total aqueous and octanol concentrations of $2,4,6-\operatorname{TrCP}$ obtained for the $64 \mathrm{mM}$ initial concentration of 2,4,6-TrCP in octanol, $\Delta\left([\mathrm{HA}]_{\mathrm{aq}}+[\mathrm{A}-]_{\mathrm{aq}}\right)$ designates the calculated maximum error. 


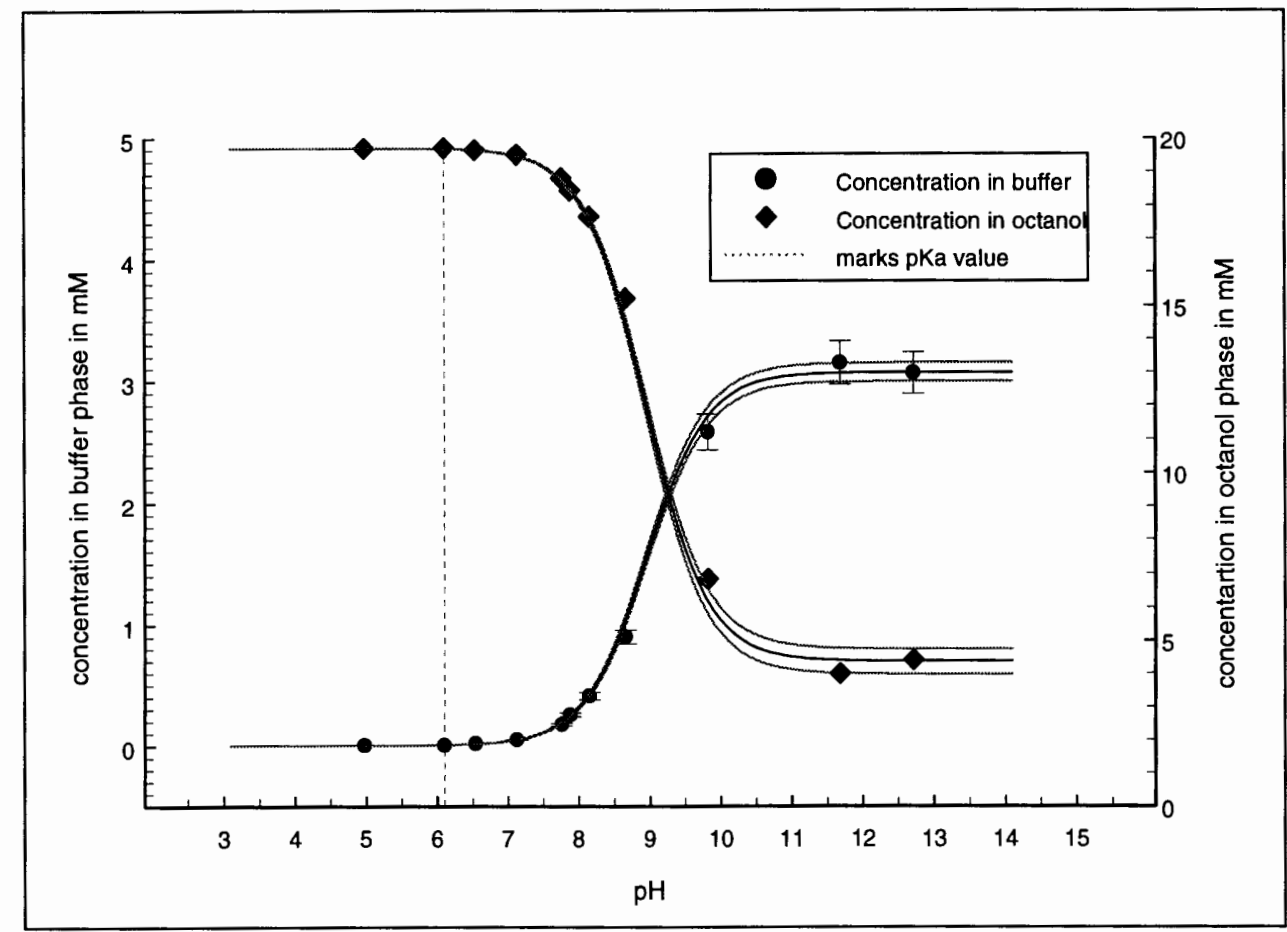

Figure 19. $\mathrm{pH}$ dependence of 2,4,6-TrCP concentrations in buffer (left axis) and octanol (right axis) obtained with $20 \mathrm{mM}$ initial octanol concentration of $2,4,6-\operatorname{TrCP}$. The solid curves illustrate prediction of the partition model for the best fit parameters. The error bars illustrate maximum experimental error. 


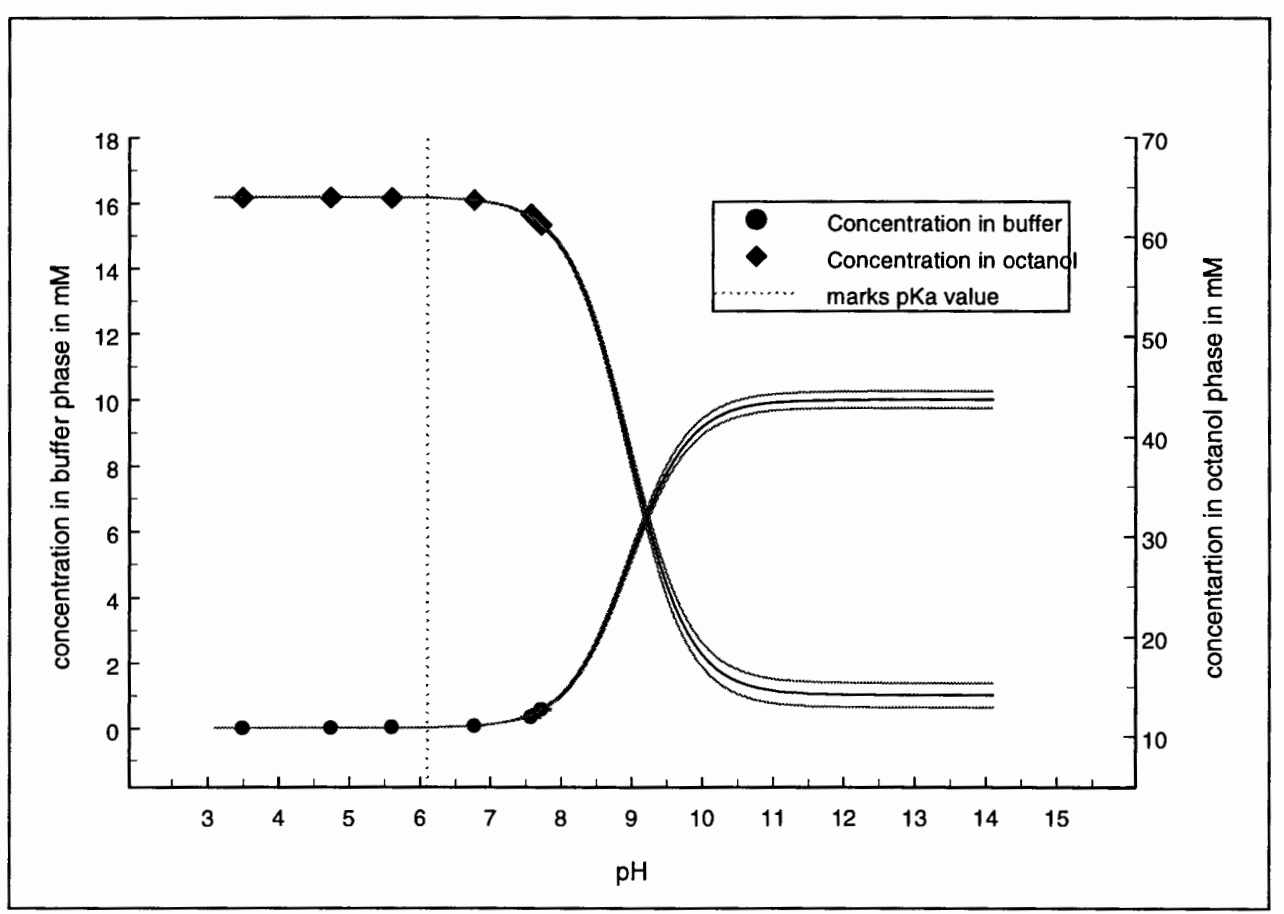

Figure 20. $\mathrm{pH}$ dependence of 2,4,6-TrCP concentrations in buffer (left axis) and octanol (right axis) obtained with $64 \mathrm{mM}$ initial octanol concentration of 2,4,6-TrCP. The solid curves illustrate prediction of the partition model for the best fit parameters. The error bars illustrate maximum experimental error. 


\section{Discussion}

\subsection{Comparison of the Octanol-Water System with the Membrane-Water System}

The knowledge of the octanol-water partition coefficient of the ionized species of 2,4,6-TrCP, $\mathrm{g}_{\mathrm{A}}$, makes it possible to compare its value with that obtained for the lipid membrane water system. Blochel (1994) measured the linear partition coefficient, $\beta_{\mathrm{A}}$, of ionized 2,4,6-TrCP for lipid membranes and obtained, $\beta_{\mathrm{A}}=$ $(6.55 \pm 0.33) \cdot 10^{-7} \mathrm{~m}$. The linear partition coefficient $\beta$ can be related to the octanol-water partition coefficient after transformation using Eq. 58, to obtain the bulk partition coefficient of ionized species for lipid membranes $\gamma_{\mathrm{A}}$. Using a membrane thickness of $\mathrm{t}=3.8 \mathrm{~nm}$ (McIntosh and Simon, 1986) one obtains then a bulk partition coefficient $\gamma_{\mathrm{A}}=(3.45 \pm 0.17) \cdot 10^{2}$. The value of $\gamma_{\mathrm{A}}$ is about 240 times greater than that predicted by the octanol-water partition coefficient. Similar behavior was found for 2,3,4,5-Tetrachlorophenol which was studied by Schmidt (1995) using the same experimental methods and for pentachlorophenol (Smejtek and Wang, 1993). The partition coefficients of these three different chlorophenols are shown in Table 6. 


\begin{tabular}{|c|c|c|c|c|c|}
\hline $\begin{array}{c}\text { molecular } \\
\text { species }\end{array}$ & $\log \gamma_{\mathrm{A}}{ }^{\mathrm{a}}$ & $\log \mathrm{g}_{\mathrm{A}}$ & $\frac{\log \mathrm{g}_{\mathrm{A}}}{\log \gamma_{\mathrm{A}}}$ & $\log \mathrm{g}_{\mathrm{HA}}$ & $\frac{\log \mathrm{g}_{\mathrm{HA}}}{\log \gamma_{\mathrm{A}}}$ \\
\hline $2,4,6-\mathrm{TrCP}$ & $2.538 \pm 0.022$ & $0.15 \pm 0.05$ & 0.0591 & $3.65 \pm 0.02$ & 1.44 \\
\hline $2,3,4,5-\mathrm{TeCP}$ & $3.475 \pm 0.015$ & $0.87 \pm 0.10^{\mathrm{b}}$ & 0.250 & $4.52 \pm 0.05^{\mathrm{b}}$ & 1.30 \\
\hline PCP & $4.281 \pm 0.02$ & $1.48^{\mathrm{c}}$ & 0.346 & $5.08^{\mathrm{d}}$ & 1.19 \\
\hline
\end{tabular}
a: Blochel (1994)
b: Schmidt (1995)
c: in $0.1 \mathrm{M} \mathrm{KCl} \mathrm{(Jafvert} \mathrm{and} \mathrm{Westall,} \mathrm{1990)}$
d: Xie (1984)

Table 6. Comparison of bulk partition coefficients of three different chlorophenols for lipid-membrane-water and octanol-water systems.

The following conclusions can be made:

(a) The lipid-membrane-water partition coefficient $\gamma_{\mathrm{A}}$ is always several orders of magnitude greater than the octanol-water partition coefficient $\mathrm{g}_{\mathrm{A}}$. In view of this result the octanol-water partition coefficient is not a suitable tool for the prediction of distribution of the ionized form of the above chlorophenols between water and lipid membranes. It is to be expected that the octanol-water partition coefficient $\mathrm{g}_{\mathrm{A}}$ seriously underestimate the concentration of ionized chlorophenols in biological membranes.

(b) The partition coefficients for the membrane-water system as well as for the octanol-water system increase with the number of chlorine substituents on the benzene ring. 
The differences of the partition coefficients of the membrane-water system and the octanol-water system can be based on the physical properties of the two model systems. The main difference is that octanol, as a bulk phase, requires maintainance of the electroneutrality and therefore co-partitioning of counterions which requires additional free energy. A description of the partition mechanism in the octanol-water system is given in Appendix B. 


\section{Appendix A}

\section{Derivations: The Gouy-Chapman Theory}

Consider an infinite uniformly charged surface in contact with an infinite homogenous electrolyte. Ions in an electric field obey a Boltzmann distribution, Eq. 1, and cause a non-vanishing space charge density, Eq. 3. This in turn gives rise to an electric potential, Eq. 2. A combination of Eqs. 1, 2 and 3 results in the Poisson-Boltzmann equation

$$
\frac{\mathrm{d}^{2} \psi(\mathrm{z})}{\mathrm{dz}^{2}}=-\frac{1}{\varepsilon_{\mathrm{r}} \varepsilon_{0}} \sum_{\mathrm{i}} \mathrm{z}_{\mathrm{i}} \mathrm{eN} \mathrm{N}_{\mathrm{i}}(\infty) \exp \left(-\frac{\mathrm{z}_{\mathrm{i}} \mathrm{e} \psi(\mathrm{z})}{\mathrm{kT}}\right)
$$

The Poisson-Boltzmann equation is a nonlinear differential equation which has to be, in general, solved numerically. A first integration of Eq. 4 is analytically possible by use of the following transformation:

Multiplication of each side of Eq. 4 by $2 \frac{\mathrm{d} \psi}{\mathrm{dz}}$ and the use of the identity $2 \frac{\mathrm{d} \psi}{\mathrm{dz}} \frac{\mathrm{d}^{2} \psi}{\mathrm{dz}^{2}}=\frac{\mathrm{d}}{\mathrm{dz}}\left(\frac{\mathrm{d} \psi}{\mathrm{dz}}\right)^{2}$ transforms Eq. 4 to

$$
\frac{\mathrm{d}}{\mathrm{dz}}\left(\frac{\mathrm{d} \psi}{\mathrm{dz}}\right)^{2}=-2 \frac{\mathrm{d} \psi}{\mathrm{dz}} \frac{1}{\varepsilon_{\mathrm{r}} \varepsilon_{0}} \sum_{\mathrm{i}} \mathrm{z}_{\mathrm{i}} \mathrm{eN} \mathrm{N}_{\mathrm{i}}(\infty) \exp \left(-\frac{\mathrm{z}_{\mathrm{i}} \mathrm{e} \psi(\mathrm{z})}{\mathrm{kT}}\right)
$$


The Poisson-Boltzmann equation in this form Eq. 1a can then be easily integrated from $\mathrm{z}$ to infinity.

$$
\left.\left(\frac{\mathrm{d} \psi}{\mathrm{dz}}\right)^{2}\right|_{\mathrm{Z}} ^{\infty}=-\left.\frac{2 \mathrm{kT}}{\varepsilon_{\mathrm{r}} \varepsilon_{0}} \sum_{\mathrm{i}} \mathrm{N}_{\mathrm{i}}(\infty) \exp \left(-\frac{\mathrm{z}_{\mathrm{i}} \mathrm{e} \psi(\mathrm{z})}{\mathrm{kT}}\right)\right|_{\mathrm{Z}} ^{\infty}
$$

The boundary conditions are

$$
\lim _{\mathrm{z} \rightarrow \infty} \frac{\mathrm{d} \psi}{\mathrm{dz}}=0 \quad \text { and } \quad \lim _{\mathrm{z} \rightarrow \infty} \psi(\mathrm{z})=0
$$

This results in the nonlinear equation

$$
\left(\frac{\mathrm{d} \psi}{\mathrm{dz}}\right)^{2}=\frac{2 \mathrm{kT}}{\varepsilon_{\mathrm{r}} \varepsilon_{0}} \sum_{\mathrm{i}} \mathrm{N}_{\mathrm{i}}(\infty)\left[\exp \left(-\frac{\mathrm{Z}_{\mathrm{i}} \mathrm{e} \psi(\mathrm{z})}{\mathrm{kT}}\right)-1\right] .
$$

The double layer consisting of the membrane surface charge and the space charge of opposite polarity in the aqueous phase have to be, as a whole, electrically neutral. Consequently, the charge on the surface of the membrane must be balanced by the charge in the solution; i.e.

$$
\sigma_{\mathrm{m}}=-\int_{0}^{\infty} \rho(\mathrm{z}) \mathrm{dz},
$$

$\sigma_{\mathrm{m}}$, denotes the surface charge density on the membrane surface. By substituting for $\rho(\mathrm{z})$, Eq. 2, into Eq. 5a we obtain 


$$
\sigma_{\mathrm{m}}=\int_{0}^{\infty} \varepsilon_{\mathrm{r}} \varepsilon_{0} \frac{\mathrm{d}^{2} \psi(\mathrm{z})}{\mathrm{dz}} \mathrm{dz}
$$

which together with the boundary condition $\lim _{\mathrm{z} \rightarrow \infty} \frac{\mathrm{d} \psi}{\mathrm{dz}}=0$, can be integrated to yield

$$
\sigma_{\mathrm{m}}=-\left.\varepsilon_{\mathrm{r}} \varepsilon_{0} \frac{\mathrm{d} \psi(\mathrm{z})}{\mathrm{dz}}\right|_{\mathrm{z}=0}
$$

The combination of Eqs. $4 \mathrm{a}$ and $7 \mathrm{a}$ results in the Gouy-Chapman equation,

$$
\sigma_{m}= \pm\left[2 \varepsilon_{r} \varepsilon_{0} k T \sum_{i} N_{i}(\infty)\left\{\exp \left(-\frac{z_{i} e \psi(0)}{k T}\right)-1\right\}\right]^{\frac{1}{2}}
$$

where $\psi(0)$ is the potential at the membrane surface. 


\section{Appendix B}

\section{The Partitioning in the Octanol-Water System: Multicomponent Partitioning}

In the ideal partitioning theory, section 4.1.1, we assumed that the partitioning of a species depends on the free energy of tranfer of this particular species and we neglected any interactions between different species. This idealization can not be applied to the partitioning of the ionized species. The partitioning of the ionized species is strongly coupled to the partitioning of counterions, in our experiment to potasssium ions, $\mathrm{K}^{+}$, to satisfy the electroneutrality of the two phases. The partitioning of $\mathrm{A}^{-}$has to be treated as a multicomponent chemical equilibrium problem rather than a single component equilibrium problem (Westall et al., 1985). The following reactions between the octanol and water bulk phases can qualitatively describe the partitioning of $2,4,6-\operatorname{TrCP}$, as suggested by Westall.

(i) Solvation of the neutral organic compound in bulk octanol phase :

$$
(H A)_{a q} \leftrightarrow(H A)_{o c}
$$

(ii) Solvation of free ions or ion pairs in the bulk octanol phase : 


$$
\begin{array}{ll}
K_{a q}^{+}+A_{a q}^{-} \leftrightarrow K_{o c t}^{+}+A_{o c I}^{-} & \mathrm{C}_{\mathrm{I}} \\
K_{a q}^{+}+A_{a q}^{-} \leftrightarrow(K A)_{o c I I} & \mathrm{C}_{\mathrm{II}}
\end{array}
$$

where $(\mathrm{HA})_{\mathrm{aq}}, \mathrm{K}_{\mathrm{aq}}^{+}$and $\mathrm{A}_{\mathrm{aq}}^{-}$are the species in the aqueous phase, and (HA) $)_{\mathrm{oc}}$, $\mathrm{K}_{\text {ocI }}^{+},(\mathrm{KA})_{\text {ocII }}$ and $\mathrm{A}_{\text {ocI }}^{-}$are the species in the octanol phase. $\mathrm{C}_{\mathrm{I}}$ and $\mathrm{C}_{\mathrm{II}}$ are the dissociation coefficients of reaction I and II.

Reaction (i) describes the partitioning of the neutral species, which is an ideal partitioning process. The reactions suggested in (ii) show the dependence of $\mathrm{A}^{-}$-partitioning on the ionic strength. In principal, the partitioning of $\mathrm{A}^{-}$ increases with the increasing ionic strength. The reactions (ii) are the most probable reactions to describe the sorption of the ionized form of 2,4,6-TrCP to the octanol phase. But sorption of $\mathrm{A}^{-}$can also occur as adsorption ${ }^{1}$ of $\mathrm{A}^{-}$to the octanol, with a counterion in the electric double layer, or as a ligand exchange of the organic molecule with a surface hydroxyl group at the inorganic oxide surface. Those reactions which take place at the interface are thought to play a minor role in the sorption process as octanol and water are used as extended bulk phases.

Using the dissociation coefficients of the reactions in (ii),

$$
\begin{gathered}
C_{I}=\frac{\left[K^{+}\right]_{o c L}\left[A^{-}\right]_{o c I}}{\left[K^{+}\right]_{a q}\left[A^{-}\right]_{a q}} \Rightarrow \frac{\left[A^{-}\right]_{o c I}}{\left[A^{-}\right]_{a q}}=\frac{\left[K^{+}\right]_{a q}}{\left[K^{+}\right]_{o c I}} \cdot C_{I} \\
C_{I I}=\frac{[(K A)]_{o c I I}}{\left[K^{+}\right]_{a q}\left[A^{-}\right]_{a q}} \Rightarrow \frac{\left[A^{-}\right]_{o c I I}}{\left[A^{-}\right]_{a q}}=\left[K^{+}\right]_{a q} \cdot C_{I I}
\end{gathered}
$$

\footnotetext{
${ }^{1}$ This is an adsorption mechanism like in the membrane-water system.
} 
$\mathrm{g}_{\mathrm{A}}$ can be expressed as

$$
g_{A}=\frac{\left[A^{-}\right]_{o c}}{\left[A^{-}\right]_{a q}}=\left[K^{+}\right]_{a q} \cdot\left\{\frac{C_{I}}{\left[K^{+}\right]_{o c I}}+C_{I I}\right\}
$$

Equation $1 \mathrm{~b}$ shows the nonlinear dependence of $\mathrm{g}_{\mathrm{A}}$ on the potassium concentration in the aqueous phase. The partition coefficient $g_{A}$ increases with increasing potassium concentration. $\mathrm{C}_{\mathrm{I}}$ and $\mathrm{C}_{\mathrm{II}}$ are characteristic constants for the transport of $\mathrm{K}^{+}$and $\mathrm{A}^{-}$, either as free ions (reaction $\mathrm{I}$ ) or as ion pairs (reaction II), from the aqueous to the octanol phase Experimental data from Schwarzenbach $^{2}(1985)$ predicted the existence of the free ions rather than ion pairs in the octanol phase. If we consider that this is also applicable to our system

$$
\left[K^{+}\right]_{o c}=\left[A^{-}\right]_{o c}=\left(\left[A^{-}\right]_{a q}\left[K^{+}\right]_{a q} C_{I}\right)^{1 / 2}
$$

the expression for $g_{A}$ can be simplified to

$$
g_{A}=\frac{\left[A^{-}\right]_{o c}}{\left[A^{-}\right]_{a q}}=\left(\frac{\left[K^{+}\right]_{a q}}{\left[A^{-}\right]_{a q}} \cdot C_{I}\right)^{1 / 2}
$$

\footnotetext{
${ }^{2}$ Schwarzenbach measured the octanol-water distribution ratio of $\mathrm{PCP}$ and $2,3,4,5 \mathrm{TeCP}$ at $\mathrm{pH} \sim 12$ as a function of the ratio $\left[\mathrm{Na}^{+}\right] /\left[\mathrm{A}^{-}\right]$and found linear relationship between $\log \mathrm{g}_{\mathrm{A}}$ and $\left[\mathrm{Na}^{+}\right] /[\mathrm{A}]$.
} 
The important feature of either case, $\mathrm{C}_{\mathrm{II}}=0$ or $\mathrm{C}_{\mathrm{II}} \neq 0$, is that the free energy of transfer of $\mathrm{A}^{-}$-ions from the aqeous to the octanol phase depends on the energy needed not only to carry an $\mathrm{A}^{-}$-ion but also $\mathrm{K}^{+}$-ion across the interface. $\log \left(g_{A}\right)$, which is proportional to the free energy of transfer, is therefore a function of the ionic strength in the aqueous phase. In view of this non-ideal behaviour the partitioning of $\mathrm{A}^{-}$cannot be treated according to an ideal partitioning theory. 


\section{References}

Aveyard, R.and D.A. Haydon. 1973. An introduction to the principles of surface chemistry. Cambridge University Press, Cambridge.

Beynon, K.I., D.G. Crosby, F. Korte, G.G. Still, J.W. Vonk, P.A. Greve. Environmental chemistry of pentachlorophenol. Pure and Appl. Chem. 53:1051-1080.

Blochel, A. 1992. Adsorption of halogenated phenolate ions to eggphosphatidylcholine vesicles. Master's thesis, Portland State University.

Bruijn, J. and J. Hermens. 1990. Relationships between octanol/water partition coefficients and total molecular surface area and total molecular volume of hydrophobic organic materials. Quant. Struct.-Act. Relat. 9:11-21.

Callahan, M.A., M.W. Slimak, N.W. Gabel, I.P. May, C. Fowler, J.R. Freed, P. Jennings, R.L. Durfee, F.C. Whitmore, B. Maestri, W.R. Maybe, B.R. Holt, C. Gould. 1979. Water-Related Environmental Fate of 129 Priority Pollutants. U.S. Environmental Protection Agency: Washington, DC. Vol. I and II, EPA440/4-79-029a and EPA-440/4-79-029b. 
Chessells, M., D.W. Hawker, D.W. Connell. 1991. Critical evaluation of the measurement of the 1-octanol/water partition coefficient of hydrophobic compounds. Chemosphere 22:1175-1190.

Chiou, C.T., D.W. Schmedding, M. Mannes. 1982. Partitioning of organic compounds in octanol-water systems. Environ. Sci. Technol. 16:4-10.

Gerthsen, C, H.O. Kneser, H. Vogel. 1986. Physik: Ein Lehrbuch zum Gebrauch neben Vorlesungen. Springer Verlag, Berlin Heidelberg.

Geyer, H.J., I. Scheunert, F. Korte. 1987. Distribution and bioconcentration potential of the environmental chemical pentachlorophenol (PCP) in different tissues of humans. Chemosphere 16:887-899.

Hattemeyer-Frey, H., C.C. Travis. 1989. Pentachlorophenol: Environmental partitioning and human exposure. Arch. Environ. Contam. Toxicol. 18:482-489.

Herrmann F. 1993. Physik V, Thermodynamik. Universität Karlsruhe. Skripten zur Experimentalphysik.

Jafvert, C. and J.C. Westall. 1990. Distribution of hydrophobic ionogenic organic compounds between octanol and water: Organic acids. Environ. Sci. Technol. 24: 1795-1803.

Lagas, P. 1988. Sorption of chlorophenols in the soil. Chemosphere 17:205216. 
Leo, A., C. Hansch, D. Elkins. 1971. Partition coefficientsand their uses. Chem. Rev. 71:525-616.

Mackay, D. 1982. Correlation of bioconcentration factors. Environ. Sci. Technol. 16:274-278.

Miller, M.M., S.P. Wasik. 1985. Relationships between octanol-water partition coefficient and aqueous solubility. Environ. Sci. Technol. 19:522-529.

Mussalo-Rauhamaa, H., H. Pyysalo, K. Antervo. 1989. The presence of chlorophenols and their conjugates in finnish human adipose and liver tissue. The Science of Total Environment 83:161-172.

Nelson, A.P. and D.A.McQuarrie. 1975. The effect of discrete charges on the electrical properties of a membrane I. J. theor. Biol. 55:13-27.

Nicholson, D.K., S.L. Woods, J.D. Istok, D.C. Peek. 1992. Reductive dechlorination of chlorophenols by a pentachlorophenol-acclimated methanogenic consortium. Applied and Environmental Microbiology :22802286.

OECD, Organisation of Economic Co-operation and Development. 1981. Partition Coefficients. In: Guidelines for testing of Chemicals, 107, Paris, France. 
Paasivirta, J., H. Tenhola, H. Palm, R. Lammi. 1992. Free and bound chlorophenols in kraft pulp bleaching effluents. Chemosphere 24:1253-1258.

Sabijic, A., H. Güsten, J. Hermens, A. Opperhulzen. 1993. Modeling octanol/water partition coefficients by molecular topology: Chlorinated Benzenes and Biphenyls. Environ. Sci. Technol. 27:1394-1402.

Schwarzenbach, R.P, W. Giger, C. Schaffner, O. Wanner. 1985. Groundwater contamination by volatile halogenated alkanes: Abiotic formation of volatile sulfur compounds under anaerobic conditions. Environ. Sci. Technol. 19:322327.

Schellenberg, K., C.Leuenberg, R.P. Schwarzenbach. 1984. Sorption of Chlorinated Phenols by Natural Sediments and Aquifer Materials. Environ. Sci. Technol. 18:652-657.

Schmidt, P.O. 1995. Origins of effective charge of multivalent ions at a membrane/water interface and distribution of 2,3,4,5-Tetrachlorophenol in a membrane model system. Master's thesis, Portland State University.

Silverstein, R.M., G. C. Bassler, T.C. Morrill. 1991. Spectrophotometric identification of organic compounds. John Wiley \& Sons.

Singer, S.J. and G.L. Nicolson. 1972. The fluid mosaic model of the structure of cell membranes. Science 175:720-731. 
Sittig, M. 1985. Handbook of Toxic and Hazardous Chemicals and Carcinogens. Noyes Publications, Park Ridge, N.J.

Smejtek, P., K. Hsu and W.H. Perman. 1976. The electrical conductivity in lipid bilayer membranes induced by pentachlorophenol. Biophys. J. 16:319336.

Smejtek, P. and S. Wang. 1993. Distribution of hydrophobic ionizable xenobiotic between water and lipid membranes: Pentachlorophenol and pentachlorophenate. Arch. Environ. Contam. Toxicol. 25:394-404.

Stryer, L. 1981. Biochemistry. W.H. Freeman and Company, San Francisco.

Terada, H. 1990. Uncouplers of oxidative phosphorylation. Environmental Health Perspectives 87:213-218.

Völker, D. 1994. Interaction of Ruthenium Red with Phospholipid vesicles. Master's thesis, Portland State University.

Wang,L., R. Govind, R.A. Dobbs. 1993. Sorption of toxic organic compounds on wastewater solids: Mechanism and modeling. Environ. Sci. Technol. 27:152-158.

Westall, J.C., C. Leuenberger, R.P. Schwarzenbach. 1985. Influence of $\mathrm{pH}$ and Ionic Strength on the Aqueous-Nonaqueous Distribution of Chlorinated Phenols. Environ. Sci. Technol. 19:193-198. 
Westall, J.C., C.A. Johnson, W. Zhang. 1990. Distribution of $\mathrm{LiCl}, \mathrm{NaCl}, \mathrm{KCl}$, $\mathrm{HCl}, \mathrm{MgCl}_{2}$, and $\mathrm{CaC}_{2}$ between Octanol and Water. Environ. Sci. Technol. 24:1803-1810.

Wild, S.R., S.J. Harrad, K.C. Jones. 1992. Pentachlorophenol in the UK environment I : a budget and source inventory. Chemosphere 24:833-845.

Wild, S.R., K.C. Jones. 1992. Pentachlorophenol in the UK environment II: Human exposure and an assessment of pathways. Chemosphere 24:847-855.

Xie, T.M., B. Hulthe, S. Folestad. 1984. Determination of partition coefficients of chlorinated phenols, guaiacols and catechols by shake-flask GC and HPLC. Chemosphere 13:445-459. 